\title{
Caracterização dos Recifes de Corais da Área de Preservação Ambiental da Baía de Todos os Santos para Fins de Manejo, Bahia, Brasil *
}

\section{Characterization of Coral Reefs from Todos os Santos Bay Protected Area for Management Purpose, Bahia, Brazil}

\author{
I. C. S. Cruz ${ }^{1,2,3}$, Kikuchi, R. K. P. ${ }^{3}$, Z. M. A. N. Leão ${ }^{3}$
}

\section{RESUMO}

A Baía de Todos os Santos (BTS) constitui uma Área de Preservação Ambiental (APA), área protegida a qual equivale à categoria $\mathrm{V}$ da International Union for Conservation of Nature, dentro da zona urbana da cidade do Salvador, na costa do estado da Bahia, a região de maior diversidade de corais do oceano Atlântico Sul Ocidental. Esta área protegida não possui, ainda, um plano de manejo, ferramenta que limita e regulariza o uso dos recursos de maneira a promover a conservação do meio ambiente. Os recifes da BTS estão dispostos em duas regiões com distintos regimes hidrodinâmicos e impactos gerados pela atividade humana. Um grupo de recifes localiza-se na entrada da baía (Externos), diretamente expostos à ação das ondas, e o outro grupo está situado no interior da baía (Internos), sob efeito de uma menor energia hidrodinâmica. O grupo dos recifes internos está mais próximo da cidade do Salvador, com 2,8 milhões de habitantes, e de um pólo industrial. Atualmente o despejo dos efluentes domésticos e industriais, diminuiu, embora não o suficiente para afirmar que houve melhoria na qualidade ambiental. Uma área de proteção ambiental, com um eficiente plano de manejo, poderá ser uma importante ferramenta legal para esta finalidade. Este trabalho tem como objetivo verificar se as diferenças existentes entre os dois grupos de recifes, quanto à comunidade dos organismos macrobentônicos recifais e a

1 autor correspondente: igorcruz@ gmail.om

2 U niversidade Federal da Bahia, Instituto de Biologia, Programa de Pós-graduação em E cologia e Biomonitoramento, Rua Barão de Jeremoabo s/ n Campus U niversitário de 0 ndina - CE P 40170-290 Salvador, Bahia, Brasil.

3 U niversidade Federal da Bahia, Instituto de G eociências, G rupo de Pesquisa em Recifes de C orais e M udanças G lobais, Rua Barão de Jeremoabo, s/ $\mathrm{n}$ - Federação - CE P 40170-290 - Salvador, Bahia, Brasil. E-mails: kikuchi@ ufba.br, Z elinda@ufba.br. 


\author{
Cruz, Kikuchi e L eão \\ Revista de G estão C osteira Integrada / Journal of Integrated C oastal Z one M anagement 9(3):3-23 (2009)
}

comunidade dos corais, justificam a criação de áreas de exclusão de uso, descrita como zona de preservação da vida silvestre em seu decreto, distintas para cada grupo. Os dados foram levantados aplicando-se a técnica do vídeo-transecto. Devido à limitação desta técnica em identificar espécies com baixa abundância, a amostragem foi complementada com a identificação visual, em campo, de todas as espécies de corais presentes em cada recife. As diferenças entre os grupos recifais foram testadas com a Análise de Similaridade, quantificadas com a Análise de Percentual de Similaridade e representadas em gráficos de Escalonamento Multidimensional. Os resultados mostram que os recifes externos e internos são diferentes quanto à comunidade dos organismos macrobentônicos e a comunidade dos corais. Os recifes localizados na entrada da baía diferenciam-se pela abundância das algas calcárias incrustantes e articuladas, e as espécies de corais mais abundantes são as do complexo Siderastrea, M ussismilia hispida, M . braziliensis e Porites branneri. Os recifes localizados no interior da baía possuem maior abundância de esponjas e corais, e a espécie de coral dominante é Montastraea cavernosa e o hidrocoral Millepora alcicornis, assim como as espécies do complexo Siderastrea ocorrem com certa abundância. Essas diferenças são marcantes e justificam a criação de áreas de exclusão de uso distintas para proteger as peculiaridades de cada um dos grupos de recifes. Os resultados deste trabalho foram repassados, como sugestão, à equipe que está elaborando o plano de manejo da APA, juntamente com a indicação de criação de duas zonas de preservação da vida silvestre.

\title{
ABSTRACT:
}

Todos os Santos Bay is a Brazilian E nvironmental Protection A rea equivalent to C ategory V of the International U nion for C onservation of $\mathrm{N}$ ature, which endoses two groups of oral reefs in the region of the greatest biodiversity in the $\mathrm{W}$ estern South $\mathrm{A}$ tlantic $\mathrm{O}$ can, the coast of the state of Bahia. This type of Protected A rea aims the sustainable use of the natural resouroes and the biodiversity conservation. The Todos os Santos Bay E nvironmental Protection A rea does not have a management plan, yet, that is a tool for restricting the use of natural resouros in order to promote conservation of the environment. In Todos os Santos Bay there are two regions of reefs with different hydrodynamic regimes and human impacts: the reefs located at the entrance of the bay, the outside reefs, which are directly ex posed to the wave's action, and the largest group of reefs that is located inside the bay in a lower hydrodynamic regime. These later reefs are located near Salvador, a city with 2.8 million inhabitants and of an industrial center, with a history of more than 40 years of pollution. $\mathrm{N}$ owadays, dumping of both domestic and industrial wastewater has decreased, although much still needs to be improved, and the area of environmental protection can be an important legal tool to this purpose. This study aims to determine whether the differ ences between these two groups of reefs regarding the macrobentonic and coral communities justify the creation of an independent $\mathrm{N} 0 \mathrm{~T}$ ak e Z one for each group. T wenty three stations were sampled, eight in the outside reefs and fitteen in the inside reefs, all during scuba diving, applying the video-transect technique, along six transects per reef station, each 20 $\mathrm{m}$ long and $0.21 \mathrm{~m}$ wide. D ue to limitation of this technique for identifying species with low abundanoe, sampling was omplemented with visual identification of all species of corals in each reef station. The videe-transect images were analyzed in the free software V ITRA with 20 points per frame. Three matrioes were generated, one of the macrobentonic community structure, the second one of the coral community structure and one third of the coral community composition. The qualitative matrix generated by the visual identification of orals was better for the coral species distribution than the quantitative one generated by the video-transect. D ifferenoes between the two reef groups were tested with the analysis of similarity. In order to quantify the differenoes it was used the similarity percentage, and for the graph representation the multidimensional scaling. Because the matrioes of the macrobentonic and the coral community structures are compose of the same set of data, generated by the analysis of the video-transects, the alpha was adjusted for Bonferroni orrection from 0.050 to 0.025 . H owever, for the oral community composition, the alpha was maintained in 0.050 because these data come from the visual identification in the field. The results were significant for both, the oral community structure and the oral community composition. The refs located at the entrance of the bay are differentiated by the highest abundanœ of the incrusted and articulated calcareous algae. The most abundant species of orals are the ones belonging to the complex Siderastrea and the species M ussismilia hispida, M . braziliensis and Porites branneri. The reefs located at the interior of the bay have a higher abundance of corals and sponges. The dominant coral specie is M ontastraea cavernosa, but the hydrooral M illepora alcicornis and the Siderastrea complex are very abundant as well. These differenoes are significant and justify the creation of two independent no take zones in order to protect the peculiarities of each reef group. The results of this study were given as suggestions to the team that is preparing the Todos os Santos Bay management plan, along with the suggestion for creating two $\mathrm{N} 0 \mathrm{~T}$ ake Z ones.

\section{INTRODUÇÃO}

A criação de Áreas de Exclusão de Uso (AEUs), pesca e outras atividades humanas, é proposta como o melhor instrumento contra a degradação ambiental por impactos locais (Hodgson, 1999; Souter \& Lindén, 2000; Bellwood et al., 2004; Aronson \& Precht, 2006; McClanahan et al., 2006; Mumby, 2006). O efeito da exclusão do uso leva à recuperação e 
manutenção da integridade ecológica (Edmmunds \& Carpenter, 2001) e confere, ao ecossistema, uma maior capacidade de resistir e recuperar-se (resiliência) de impactos globais, a exemplo do problema das mudanças climáticas que tem como um dos seus efeitos o fenômeno de branqueamento dos corais (Pandolfi et al., 2003; West \& Salm, 2003; Bellwood et al., 2004). Isto ocorre por meio da recuperação e estabilização das populações de grupos funcionais, a exemplo dos peixes herbívoros de grande porte e ouriços, que controlam as algas frondosas, fortes concorrentes dos corais (Edmmunds \& Carpenter, 2001; West \& Salm, 2003; Bellwood et al., 2004). Estima-se que pelo menos $20 \%$ dos recifes de corais devam constituir AEUs para garantir sua conservação (Souter \& Lindén, 2000; Halpern, 2003; Bellwood et al., 2004; Wilkinson, 2004). Por este motivo o Parque da Grande Barreira de Corais da Austrália ampliou suas AEUs de 4,5\% para 33\% de sua área (Wilkinson, 2004; Fernandes et al., 2005).

É atribuído também às AEU's o aumento em número, tamanho e capacidade reprodutiva dos peixes (McClanahan et al., 1999; Halpern, 2003), além da possibilidade de favorecer a melhoria de áreas adjacentes com a migração de larvas e indivíduos adultos de diversos organismos (Wantiez et al., 1997; Shanks et al., 2003; Bellwood et al., 2004). Isso foi constatado na Reserva Extrativista (RESEX) de Corumbau que vem apresentando bons resultados no aumento da biomassa de peixes comerciais com uma AEU com uma área recifal de $18,5 \mathrm{~km}^{2}$ (Moura, et al., 2007). Este fenômeno beneficiará, principalmente, as comunidades tradicionais que vivem da pesca nestes recifes.

A Área de Proteção Ambiental (APA) da Baía de Todos os Santos (BTS) apresenta-se como uma oportunidade de criação de uma ou mais AEUs. Esta APA foi criada pelo Governo do Estado da Bahia em 1999, através do decreto N 7.595 (Governo do Estado da Bahia, 1999). A APA é uma categoria de área protegida do Sistema Nacional de Unidades de Conservação brasileiro (SNUC) e é equivale á categoria V, Proteção da Paisagem, da International Union for Conservation of Nature (IUCN) (Dudley, 2008; Rylands \& Brandon, 2005; Silva, 2005a, b; Presidência da República, 2000). Esse tipo de Área Protegida tem como finalidade criar uma base legal para gerir os recursos ambientais de maneira sustentável e conservar a biodiversidade (Presidência da República, 2000). A conservação ocorre por meio do estabelecimento de regras de restrições e proibições do uso dos seus recursos, e as normas para isto devem estar contidas em seu plano de manejo (Governo do Estado da Bahia, 1999). Dentre estas regras, o decreto da APA da BTS prever a demarca de Zonas de Preservação da Vida Silvestre (ZPVS) (Governo do Estado da Bahia, 1999), as quais na parte marinha funcionariam como AEUs. Entretanto, nove anos após sua criação, a APA da BTS ainda não dispõe de seu plano de manejo. Esta condição reflete a situação da maior parte das demais APAs do estado da Bahia. A maioria das APAs estaduais não foram implementas e elas correspondem a 98,9\% das áreas protegidas pelo Governo da Bahia (Artaza-Barrios \& Schiavetti, 2007).

Importante ressaltar que penas implementar uma APA não garante efetividade na conservação. Após esta etapa, são necessários recursos para manutenção das atividades de gestão desta área protegida com uma continua fiscalização e uma equipe técnica qualificada e comprometida com a conservação (Artaza-Barrios \& Schiavetti, 2007; Assunção et al., 2003). Contudo a implementação é uma fase decisiva para o sucesso da APA, pois ela pode fornecer as ferramentas necessárias para uma boa gestão.

A Baía de Todos os Santos localiza-se na costa do estado da Bahia, a qual é caracterizada por ser a região de maior diversidade de corais do oceano Atlântico Sul Ocidental, com o maior número de espécies endêmicas do Brasil (Laborel, 1970; Castro \& Pires 2001; Leão et al., 2003). Os recifes da baía estão concentrados em duas regiões. Os recifes do interior da baía, localizados mais próximos do complexo industrial e da cidade do Salvador, expostos aos impactos provenientes da contaminação industrial, e os recifes externos localizados na entrada da baía, expostos a ação das águas oceânicas. Devido sua localização, esses recifes enfrentam diferentes efeitos da ação hidrodinâmica, um dos principais determinantes da estrutura das comunidades de coral (Reigl \& Piller, 1999; Madin, 2005). Na região externa da baía há uma maior intensidade de ondas e correntes de marés que na área interna da baía, Esta diferença da hidrodinâmica das duas áreas foi constatada por 


\section{Cruz, Kikuchi e L eão \\ Revista de G estão C osteira Integrada / Journal of Integrated C oastal Z one M anagement 9(3):3-23 (2009)}

Lessa et al. (2000), através do estudo do tipo e da granulométrica do sedimento do fundo da baía.

A BTS abriga, em sua margem leste, a cidade do Salvador, com 2,8 milhões de habitantes (IBGE, s/ d). Em sua margem nordeste está implantado um complexo industrial, o qual abrange metalúrgicas, fábricas de fibras sintéticas, indústrias químicas, de cerâmica, além da produção e do refino de petróleo e três portos (Tavares et al., 1999; Santos et al., 2000), todos com alto potencial de impacto ambiental. Essas atividades são responsáveis por uma série de incidentes ambientais, a exemplo do derramamento de $900 \mathrm{~kg}$ de mercúrio, na Enseada dos Tainheiros, (Wasserman \& Queiroz, 2004) e de diversos derramamentos de óleo, o maior deles despejou 48.000 litros de petróleo bruto com altas concentrações de metais e enxofre em 1992 (Orge et al., 2000), além da emissão de efluentes domésticos de uma grande parcela da cidade do Salvador (Santos et al., 2000). Diversos trabalhos evidenciam a contaminação crônica concentrada, principalmente, na região norte da baía. Foram constatados vestígios de hidrocarbonetos policíclicos aromáticos derivados de petróleo (Venturini \& Tommasi, 2004; Martins et al., 2005); contaminação por metais traço como cobre, zinco, cádmio e chumbo (Wallner-Kersanach et al., 2000; Hatje et al., 2006) e contaminação por agroquímicos proibidos como Dicloro-DifenilTricloroetano (DDT), Dicloro-Difenil-Etileno (DDE) e organoclorados (Tavares et al., 1999).

Todos esses impactos levaram a uma maior atenção da sociedade civil organizada e conseqüentemente do governo. A rede de esgoto foi ampliada, o que reduz a quantidade de efluentes domésticos despejados na BTS. As indústrias são obrigadas por órgãos governamentais a tratar e a monitorar seus efluentes, além de terem um plano de contenção de danos em caso de acidentes, o que não a eximem de multas e medidas compensatórias. Entretanto, essas melhorias não são suficientes para a recuperação e conservação da baía e o plano de manejo da APA da Baía de Todos Santos pode ser uma ferramenta decisiva para esse objetivo.

Considerando o exposto, o presente trabalho teve como objetivo caracterizar os recifes da BTS verificando se as diferenças existentes entre os recifes externos e internos da baía justificam a criação de
Zonas de Preservação da Vida Silvestre distintas. Para este propósito foram considerados a estrutura da comunidade macrobentônica dos recifes e a estrutura da comunidade dos corais. De maneira complementar, para suprir uma limitação do método do vídeotransecto (Cruz et al., 2008), foi avaliada, também, a composição da comunidade dos corais.

Questões:

$1^{\mathrm{a}}$. Os recifes internos e externos são diferentes quanto à estrutura da comunidade macrobentônica recifal?

$2^{a}$. Os recifes internos e externos são diferentes quanto à estrutura da comunidade dos corais?

$3^{a}$. Os recifes internos e externos são diferentes quanto à composição da comunidade dos corais

\section{METODOLOGIA}

\section{Área de Estudo}

Os recifes internos da BTS estão localizados ao longo da costa oeste da cidade do Salvador, bordejando o lado sudeste da ilha dos Frades e os lados leste a oeste da ilha de Maré, prolongando-se ao sul em uma série de bancos recifais entre essas ilhas e a cidade do Salvador. Os recifes externos estão localizados ao longo das costas leste e sudeste da ilha de Itaparica. Na costa leste o maior recife é o das Pinaúnas e na costa sudeste é o banco recifal das Caramuanas.

Vinte e três estações foram amostradas entre março e dezembro de 2007, sendo oito estações nos recifes internos, Pedra Alva, Pedra Cardinal, Pedra da Mangueira, Pedra do Dentão, Poste 1, Poste 4, Poste 5 e Poste 6, as mesmas estudadas por Dutra et al. (2006). A estação de Inema, localizada, também, no interior da BTS, foi selecionada a partir do trabalho de Laborel (1970). As demais quatorze estações foram selecionadas a partir de imagens de satélite, seis estão localizadas no interior da baía e oito nos recifes externos, três no banco recifal das Caramuanas e cinco no recife das Pinaunas. 


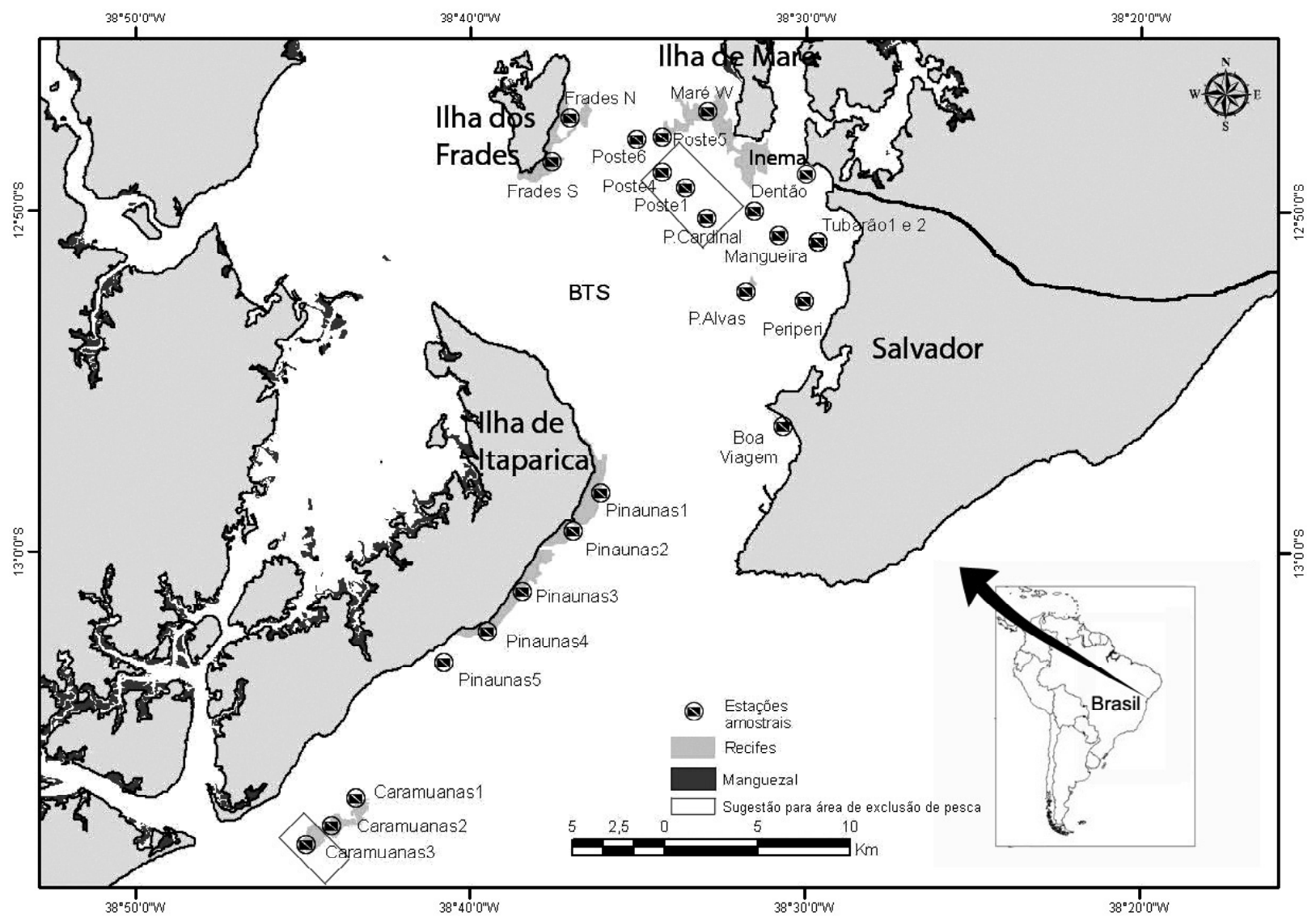

Figura 1: Localização da Baía de Todos os Santos no Brasil com indicação das estações de amostragem e sugestão de delimitação para as Zonas de Preservação da Vida Silvestre, segundo Cruz (2008).

Figure 1. L ocation of Todos os Santos Bay with indication of studied reefs with sample station and no-take area acoording to Cruz (2008).

\section{Aquisição dos dados}

Em cada estação os vídeos foram realizados ao longo de seis transectos de $20 \mathrm{~m}$ de comprimento por $0,21 \mathrm{~m}$ de largura, totalizando uma área de $4,2 \mathrm{~m}^{2}$ por transecto, e $25,2 \mathrm{~m}^{2}$ por estação. Devido ao fato dos vídeos não amostrarem as espécies com baixa abundância e à limitação na identificação de algumas espécies de corais com coralitos reduzidos (Carleton \& Done, 1995), durante a execução dos vídeos foram anotadas todas as espécies de corais observadas gerando, assim, uma matriz qualitativa. Esta matriz qualitativa foi utilizada para testar a diferença na composição da comunidade dos corais, complementando a lacuna da limitação do método quantitativo. Em todos os recifes, a amostragem foi feita entre três e oito metros de profundidade.

\section{Tratamento e análise das imagens}

As imagens foram arquivadas em DVDs. A análise dos vídeos foi realizada usando-se pontos sorteados sobre as imagens. Para essa finalidade foi utilizado um programa de código fonte livre, VITRA (http:// vitra.codigolivre.org.br/). Vinte pontos por imagem (quadro) foram sorteados, aleatoriamente, sobre uma malha de 450 possibilidades. Em média, cada transecto resultou em 82 quadros analisados. Os resultados são representados em porcentagem da cobertura dos organismos macrobentônicos e das espécies de corais, como descrito em Cruz et al. (2008). Os grupos macrobentônicos identificados foram coral, alga 
Cruz, Kikuchi e L eão

Revista de G estão C osteira Integrada / Journal of Integrated C oastal Z one M anagement 9(3):3-23 (2009)

frondosa, alga calcária incrustante, alga calcária articulada, alga filamentosa, esponja, ouriço, Palythoa caribaeorum Duchassaing e Michelotti, 1860, E pizoanthus sp., Carijoa, Z oanthus spp. e crinoide. Foram levantados ainda outros organismos não identificados e sedimento, embora estes últimos não tenham sido considerados na análise. Entre os corais foram identificadas as espécies Montastraea cavernosa Linne, 1766, M ussimilia hispida Verrill, 1868, M . harttii Verrill, 1868, M . braziliensis Verrill, 1868, Solymia wellsi Laborel, 1967, Porites astreoides Lamarck, 1807, P. branneri Rathbun 1887, A garicia agaricites Linneus, 1758, Madracis decatis Lyman, 1859 e Favia gravida Verrill, 1868, e o gênero Siderastrea Vaughan e Wells, 1943 (Neves, 2004; Neves et al., 2008), que na BTS está representado por duas espécies S. stellata Verrill, 1868 e $\mathrm{S}$. radians Pallas, 1766, as quais são difíceis de serem diferenciadas nas imagens de vídeo, e os hidrocorais Millepora acicornis Linne, 1758 e Stylaster roseus Pallas, 1766.

\section{Análise dos Dados}

Para testar as hipóteses foi utilizada a análise de similaridade não paramétrica (ANOSIM) unidirecional baseada no índice de similaridade de Bray-Curtis (Riegl \& Luke, 1998; Riegl, 1999; 2001; Clarke \& Waarwick, 2001; McField et al., 2001; Khalaf \& Kochzius, 2002). O foi ajustado para as $1^{\mathrm{a}}$ e $2^{\mathrm{a}}$ hipóteses pela correção de Bonferroni de 0,05 para 0,025 (Callegari-Jacques, 2003), porque foi utilizado o mesmo conjunto de dados. Nos casos em que a hipótese nula foi rejeitada, foi dado prosseguimento às analises utilizando-se o percentual de similaridade (SIMPER) unidirecional baseado, também, no índice de similaridade de Bray-Curtis (Clarke, 1993; Clarke \& Waarwick, 2001; McField et al., 2001; Khalaf \& Kochzius, 2002). Este procedimento tem como objetivo identificar o quanto esses grupos são diferentes através do grau de dissimilaridade. Para a melhor visualização desse padrão e suas diferenças foi aplicada a técnica multivariada, Analise de Escalonamento Multidimensional (M ulti-D imensional Scaling MDS), utilizando-se, também, o índice de similaridade Bray-Curtis (Riegl \& Luke, 1998; Riegl, 1999; 2001; Clarke e Waarwick, 2001; McField et al., 2001; Khalaf e Kochzius, 2002). Todas as análises foram feitas com o programa estatístico PRIMER 6 Beta.

\section{RESULTADOS}

Os recifes internos da BTS estão em profundidades que variaram de dois até treze metros, não aflorando na maré baixa. O sedimento perirrecifal é fino. As esponjas são muito freqüentes. A espécie de coral dominante é M ontastraea cavernosa, as espécies do complexo Siderastrea e o hidrocoral Millepora aldicornis são abundantes.

Os recifes externos afloram na maré baixa e alcançam no máximo nove metros de profundidade. O sedimento perirrecifal é predominantemente areia. Há uma grande freqüência de algas calcárias, incrustantes e ramificadas. As estações do recife das Pinaúnas apresentam uma cobertura de coral quase desprezível, diferenciando-se das estações do banco das Caramuanas. Os corais mais comuns são M ussismilia hispida, M . braziliensis e Porites branneri, e as espécies do complexo Siderastrea.

Os resultados das análises dos vídeo-transectos geraram duas matrizes, uma para a estrutura da comunidade dos grupos dos organismos macrobentônicos (Tabela 1) e outra para a estrutura da comunidade dos corais (Tabela 2). A identificação das espécies no campo gerou uma terceira matriz, a de composição da comunidade dos corais (Tabela 3). O teste ANOSIM apresentou um resultado significativo para a estrutura e para a composição da comunidade dos corais. Entretanto, o resultado não foi significativo para a estrutura da comunidade dos organismos macrobentônicos. Comtudo, o nível de significância (p) foi bem próximo do $(\square=0,025>$ $p=0,030)$. Por esse motivo, a interpretação desse resultado teve procedimento, considerando-a como marginalmente significativo. $\mathrm{E}$ as análises MDS das estações dos recifes externos e internos mostram que eles não se misturam nos três casos (Figura 2, 3 e 4). A separação entre os recifes externos e internos no gráfico MDS para a estrutura da comunidade dos organismos macrobentônicos (Figura 2) apóia a decisão de dar prosseguimento às análises desta hipótese. 
Cruz, Kikuchi e L eão

Revista de G estão C osteira Integrada / Journal of Integrated C oastal Z one M anagement 9(3):3-23 (2009)

Tabela 1: Matriz de cobertura dos grupos de organismos macrobentônicos, (X, média em \% e s, desvio padrão).

Table 1: Matrix of macrobentonics organisms cover (X, average in \% and $\mathrm{s}$, standard deviation).

\begin{tabular}{|c|c|c|c|c|c|c|c|c|c|c|c|c|c|}
\hline & & $\overline{\tilde{g}}$ & 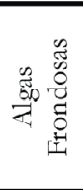 & 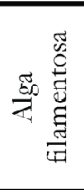 & 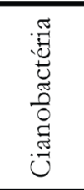 & 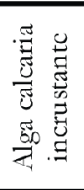 & 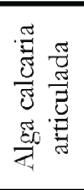 & $\begin{array}{l}\frac{\pi}{0} \\
\frac{0}{0} \\
\frac{0}{9}\end{array}$ & 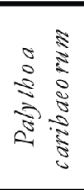 & 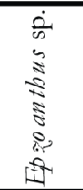 & $\begin{array}{l}\stackrel{8}{3} \\
3 \\
3\end{array}$ & 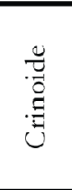 & 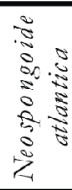 \\
\hline \multirow{2}{*}{$\begin{array}{l}\text { Caramuanas } \\
\text { Norte }\end{array}$} & $\overline{\bar{x}}$ & 6,49 & 4,39 & 22,68 & 0,00 & 34,91 & 20,49 & 0,00 & 0,00 & 0,00 & 0,04 & 0,00 & 0,00 \\
\hline & $\mathrm{s}$ & 2,83 & 2,00 & 7,90 & 0,00 & 6,05 & 5,14 & 0,00 & 0,00 & 0,00 & 0,09 & 0,00 & 0,00 \\
\hline \multirow{2}{*}{$\begin{array}{l}\text { Caramuanas } \\
\text { Leste-sueste }\end{array}$} & $\bar{x}$ & 7,42 & $0,2.3$ & 13,32 & 0,00 & 41,55 & 29,03 & 0,01 & 0,00 & 0,08 & 0,00 & 0,31 & 0,00 \\
\hline & $\mathrm{s}$ & 5,00 & 0,43 & 3,52 & 0,00 & 6,75 & 8,71 & 0,02 & 0,00 & 0,08 & 0,00 & 0,52 & 0,00 \\
\hline \multirow{2}{*}{$\begin{array}{l}\text { Caramuanas } \\
\text { Sul }\end{array}$} & $\bar{x}$ & 10,37 & 2,10 & 5,42 & 0,08 & 32,24 & 44,44 & 0,00 & 0,00 & 0,00 & 0,00 & 0,00 & 0,00 \\
\hline & $\mathrm{s}$ & 3,87 & 1,72 & 2,36 & 0,12 & 3,68 & 6,38 & 0,00 & 0,00 & 0,00 & 0,00 & 0,00 & 0,00 \\
\hline \multirow{2}{*}{ Pinaunas 1} & $\bar{x}$ & 1,12 & 5,07 & 54,72 & 13,22 & 2,50 & 16,94 & 0,28 & 0,00 & 0,00 & 0,08 & 0,29 & 0,00 \\
\hline & $\mathrm{s}$ & 0,89 & 5,90 & 15,55 & 2,32 & 0,99 & 7,79 & 0,22 & 0,00 & 0,00 & 0,20 & 0,38 & 0,00 \\
\hline \multirow{2}{*}{ Pinaunas 2} & $\bar{x}$ & 0,32 & 2,73 & 55,34 & 3,92 & 12,17 & 18,63 & 0,52 & 0,00 & 0,00 & 0,04 & 1,09 & 0,00 \\
\hline & $\mathrm{s}$ & 0,47 & 0,92 & 9,15 & 1,94 & 8,05 & 12,30 & 0,91 & 0,00 & 0,00 & 0,06 & 0,43 & 0,00 \\
\hline \multirow{2}{*}{ Pinaunas 3} & $\bar{x}$ & 0,47 & 1,12 & 54,78 & 21,10 & 8,92 & 7,99 & 0,06 & 0,00 & 0,00 & 0,11 & 0,92 & 0,00 \\
\hline & $\mathrm{s}$ & 0,60 & 1,65 & 5,88 & 12,28 & 3,16 & 9,88 & 0,08 & 0,00 & 0,00 & 0,27 & 1,13 & 0,00 \\
\hline \multirow{2}{*}{ Pinaunas 4} & $\bar{x}$ & 0,43 & 11,48 & 57,83 & 4,23 & 7,52 & 3,16 & 8,00 & 0,00 & 0,00 & 0,02 & 0,09 & 0,00 \\
\hline & $\mathrm{s}$ & 0,53 & 8,45 & 5,87 & 2,42 & 6,04 & 4,04 & 5,00 & 0,00 & 0,00 & 0,06 & 0,10 & 0,00 \\
\hline \multirow{2}{*}{ Pinaunas 5} & $\bar{x}$ & 0,21 & 7,63 & 64,33 & 0,12 & 9,11 & 0,68 & 11,01 & 0,00 & 0,00 & 0,02 & 0,27 & 0,00 \\
\hline & $\mathrm{s}$ & 0,28 & 3,21 & 2,75 & 0,25 & 5,72 & 1,05 & 5,02 & 0,00 & 0,00 & 0,05 & 0,34 & 0,00 \\
\hline \multirow{2}{*}{ Poste 1} & $\bar{x}$ & 2,35 & 0,00 & 5,31 & 0,00 & 0,02 & 0,01 & 3,82 & 0,51 & 82,70 & 0,00 & 0,00 & 0,00 \\
\hline & $S$ & 1,19 & 0,00 & 1,03 & 0,00 & 0,05 & 0,02 & 1,24 & 0,51 & 2,77 & 0,00 & 0,00 & 0,00 \\
\hline \multirow{2}{*}{ Poste 4} & $\bar{x}$ & 21,02 & 0,00 & 49,80 & 0,29 & 0,04 & 0,11 & 8,75 & 0,09 & 0,16 & 0,00 & 0,00 & 0,00 \\
\hline & $\mathrm{s}$ & 8,22 & 0,00 & 6,79 & 0,53 & 0,09 & 0,27 & 3,26 & 0,12 & 0,12 & 0,00 & 0,00 & 0,00 \\
\hline \multirow{2}{*}{ Poste 5} & $\bar{x}$ & 4,61 & 0,01 & 27,29 & 0,47 & 0,84 & 0,56 & 8,36 & 15,92 & 1,38 & 0,00 & 0,00 & 16,19 \\
\hline & $\mathrm{s}$ & 2,29 & 0,03 & 4,25 & 0,69 & 0,30 & 0,69 & 1,27 & 11,15 & 3,12 & 0,00 & 0,00 & 8,60 \\
\hline \multirow{2}{*}{ Poste 6} & $\bar{x}$ & 19,24 & 0,00 & 50,05 & 0,51 & 0,00 & 0,00 & 9,68 & 0,12 & 0,38 & 0,02 & 0,00 & 8,29 \\
\hline & $\mathrm{s}$ & 1,25 & 0,00 & 1,25 & 0,15 & 0,00 & 0,00 & 1,09 & 0,19 & 0,17 & 0,05 & 0,00 & 5,01 \\
\hline \multirow{2}{*}{$\begin{array}{l}\text { Pedra } \\
\text { Cardinal }\end{array}$} & $\bar{x}$ & 27,02 & 0,00 & 52,45 & 1,42 & 0,12 & 0,00 & 6,55 & 0,00 & 5,03 & 0,00 & 0,00 & 0,02 \\
\hline & $\mathrm{s}$ & 5,38 & 0,00 & 5,35 & 0,82 & 0,09 & 0,00 & 1,56 & 0,00 & 1,76 & 0,00 & 0,00 & 0,05 \\
\hline \multirow{2}{*}{ Pedra Alva } & $\bar{x}$ & 13,11 & 0,00 & 55,88 & 1,31 & 0,01 & 0,00 & 9,96 & 0,00 & 0,00 & 0,00 & 0,00 & 0,01 \\
\hline & $\mathrm{s}$ & 5,76 & 0,00 & 10,00 & 0,90 & 0,03 & 0,00 & 4,93 & 0,00 & 0,00 & 0,00 & 0,00 & 0,03 \\
\hline \multirow{2}{*}{$\begin{array}{l}\text { Pedta da } \\
\text { Mangueira }\end{array}$} & $\bar{x}$ & 8,39 & 0,00 & 54,34 & 0,85 & 0,00 & 0,02 & 7,56 & 0,00 & 0,01 & 0,00 & 0,00 & 1,07 \\
\hline & $\mathrm{s}$ & 4,09 & 0,00 & 17,31 & 0,97 & 0,00 & 0,06 & 2,98 & 0,00 & 0,03 & 0,00 & 0,00 & 1,66 \\
\hline \multirow{2}{*}{$\begin{array}{l}\text { Pcdra do } \\
\text { Dentão }\end{array}$} & $\bar{x}$ & 0,72 & 0,00 & 8,99 & 0,17 & 0,01 & 0,00 & 7,53 & 9,51 & 67,52 & 0,00 & 0,00 & 0,32 \\
\hline & $\mathrm{s}$ & 0,38 & 0,00 & 2,29 & 0,26 & 0,02 & 0,00 & 2,05 & 3,03 & 8,15 & 0,00 & 0,00 & 0,55 \\
\hline \multirow{2}{*}{$\begin{array}{l}\text { Ilha dos } \\
\text { Frades Leste }\end{array}$} & $\bar{x}$ & 0,87 & 43,06 & 45,46 & 0,33 & 2,43 & 0,02 & 1,29 & 0,30 & 0,00 & 0,00 & 0,00 & 0,00 \\
\hline & 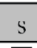 & 0,94 & 8,93 & 4,43 & 0,67 & 1,44 & 0,05 & 0,62 & 0,67 & 0,00 & 0,00 & 0,00 & 0,00 \\
\hline \multirow{2}{*}{$\begin{array}{l}\text { llha dos } \\
\text { Frades Sul }\end{array}$} & $\bar{x}$ & 13,23 & 3,54 & 13,38 & 1,06 & 0,06 & 41,90 & 5,68 & 0,00 & 0,00 & 0,00 & 0,00 & 0,09 \\
\hline & $\mathrm{s}$ & 2,74 & 1,75 & 3,07 & 0,72 & 0,12 & 6,51 & 2,71 & 0,00 & 0,00 & 0,00 & 0,00 & 0,15 \\
\hline \multirow{2}{*}{$\begin{array}{l}\text { Tha de } \\
\text { Maré }\end{array}$} & $\bar{x}$ & 3,33 & 8,93 & 30,93 & 0,14 & 0,19 & 0,75 & 7,24 & 0,42 & 0,00 & 0,00 & 0,00 & 0,00 \\
\hline & $\mathrm{s}$ & 2,11 & 12,27 & 9,16 & 0,17 & 0,29 & 0,83 & 1,83 & 0,95 & 0,00 & 0,00 & 0,00 & 0,00 \\
\hline tem & $\bar{x}$ & 6,90 & 0,00 & 34,54 & 21,51 & 0,64 & 10,94 & 6,95 & 13,07 & 0,38 & 0,13 & 0,00 & 0,00 \\
\hline gyem & $\mathrm{s}$ & 5,07 & 0,00 & 14,57 & 18,29 & 0,39 & 4,22 & 4,51 & 10,88 & 0,89 & 0,25 & 0,00 & 0,00 \\
\hline$P_{t}$ & $\bar{x}$ & 4,87 & 0,00 & 39,97 & 0,53 & 0,01 & 0,00 & 25,06 & 0,00 & 0,00 & 0,31 & 0,00 & 2,57 \\
\hline & $\mathrm{s}$ & 2,70 & 0,00 & 5,08 & 0,23 & 0,02 & 0,00 & 4,61 & 0,00 & 0,00 & 0,77 & 0,00 & 2,04 \\
\hline & $\overline{\bar{x}}$ & 1,79 & 0,00 & 19,83 & 0,57 & 0,31 & 0,00 & 20,18 & 0,04 & 4,99 & 0,28 & 0,00 & 13,73 \\
\hline 14 & 5 & 1,34 & 0,00 & 3,96 & 0,68 & 0,27 & 0,00 & 3,80 & 0,11 & 2,09 & 0,22 & 0,00 & 2,39 \\
\hline & $\bar{x}$ & 4,99 & 25,37 & 13,85 & 0,29 & 0,11 & 21,61 & 2,50 & 0,00 & 1,13 & 0,02 & 0,00 & 0,00 \\
\hline na & $\mathrm{s}$ & 2,35 & 9,53 & 9,12 & 0,18 & 0,16 & 6,37 & 1,65 & 0,00 & 2,36 & 0,05 & 0,00 & 0,00 \\
\hline
\end{tabular}


Cruz, Kikuchi e L eão

Revista de G estão C osteira Integrada / Journal of Integrated C oastal Z one M anagement 9(3):3-23 (2009)

Tabela 2: Matriz de abundância dos corais (X, média em \% e s, desvio padrão.

Table 2: Matrix of oral species abundance (X, average in $\boldsymbol{\%}$ and $\boldsymbol{s}$, standard deviation).

\begin{tabular}{|c|c|c|c|c|c|c|c|c|c|c|c|c|c|}
\hline & & 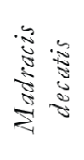 & 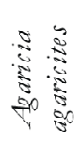 & 施 & 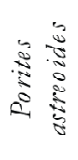 & 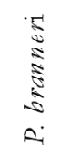 & 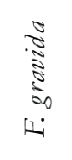 & 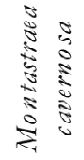 & 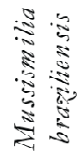 & 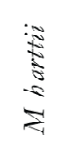 & ב- & 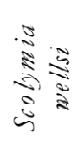 & 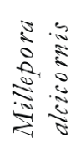 \\
\hline \multirow{2}{*}{$\begin{array}{l}\text { Caramuana } \\
\text { s Norte }\end{array}$} & $\bar{x}$ & 0,00 & 0,00 & 0,71 & 0,01 & 0,05 & 0,00 & 1,83 & 0,14 & 0,05 & 2,46 & 0,00 & 1,23 \\
\hline & $\mathrm{s}$ & 0,00 & 0,00 & 0,44 & 0,02 & 0,10 & 0,00 & 1,44 & 0,35 & 0,12 & 1,41 & 0,00 & 1,94 \\
\hline \multirow{2}{*}{$\begin{array}{l}\text { Caramuana } \\
\text { s Leste- } \\
\text { sueste } \\
\end{array}$} & $\bar{x}$ & 0,00 & 0,05 & 1,77 & 0,03 & 0,07 & 0,00 & 1,31 & 0,17 & 0,28 & 3,19 & 0,00 & 0,56 \\
\hline & $\mathrm{s}$ & 0,00 & 0,13 & 2,67 & 0,05 & 0,13 & 0,00 & 2,32 & 0,30 & 0,34 & 2,58 & 0,00 & 0,03 \\
\hline \multirow{2}{*}{$\begin{array}{l}\text { Caramuana } \\
\text { s Sul }\end{array}$} & $\bar{x}$ & 0,00 & 0,21 & 9,38 & 0,12 & 0,10 & 0,03 & 0,13 & 0,13 & 0,18 & 0,09 & 0,00 & 0,00 \\
\hline & 8 & 0,00 & 0,21 & 3,94 & 0,27 & 0,14 & 0,04 & 0,21 & 0,24 & 0,26 & 0,11 & 0,00 & 0,00 \\
\hline \multirow{2}{*}{ Pinaunas 1} & $\bar{x}$ & 0,00 & 0,00 & 0,38 & 0,00 & 0,01 & 0,00 & 0,50 & 0,11 & 0,00 & 0,13 & 0,00 & 0,00 \\
\hline & s & 0,00 & 0,00 & 0,33 & 0,00 & 0,03 & 0,00 & 0,93 & 0,27 & 0,00 & 0,15 & 0,00 & 0,00 \\
\hline \multirow{2}{*}{ Pinaunias 2} & $\bar{x}$ & 0,00 & 0,00 & 0,13 & 0,00 & 0,01 & 0,00 & 0,00 & 0,18 & 0,00 & 0,00 & 0,00 & 0,00 \\
\hline & $s$ & 0,00 & 0,00 & 0,27 & 0,00 & 0,03 & 0,00 & 0,00 & 0,40 & 0,00 & 0,00 & 0,00 & 0,00 \\
\hline \multirow{2}{*}{ Pinaunas 3} & $\bar{x}$ & 0,00 & 0,00 & 0,03 & 0,00 & 0,17 & 0,00 & 0,00 & 0,18 & 0,00 & 0,08 & 0,00 & 0,00 \\
\hline & $\mathrm{s}$ & 0,00 & 0,00 & 0,05 & 0,00 & 0,21 & 0,00 & 0,00 & 0,41 & 0,00 & 0,10 & 0,00 & 0,00 \\
\hline \multirow{2}{*}{ Pinaunas 1} & $\bar{x}$ & 0,00 & 0,00 & 0,01 & 0,00 & 0,07 & 0,00 & 0,00 & 0,05 & 0,00 & 0,31 & 0,00 & 0,00 \\
\hline & $\mathrm{s}$ & 0,00 & 0,00 & 0,03 & 0,00 & 0,11 & 0,00 & 0,00 & 0,11 & 0,00 & 0,56 & 0,00 & 0,00 \\
\hline \multirow{2}{*}{ Pinaunas 5} & $\bar{x}$ & 0,00 & 0,00 & 0,05 & 0,00 & 0,02 & 0,00 & 0,00 & 0,09 & 0,00 & 0,05 & 0,00 & 0,00 \\
\hline & 8 & 0,00 & 0,00 & 0,11 & 0,00 & 0,06 & 0,00 & 0,00 & 0,20 & 0,00 & 0,11 & 0,00 & 0,00 \\
\hline \multirow{2}{*}{ Poste 1} & $\bar{X}$ & 0,00 & 0,00 & 0,03 & 0,00 & 0,00 & 0,00 & 1,45 & 0,00 & 0,00 & 0,00 & 0,00 & 0,87 \\
\hline & $\mathrm{s}$ & 0,00 & 0,00 & 0,06 & 0,00 & 0,00 & 0,00 & 0,62 & 0,00 & 0,00 & 0,00 & 0,00 & 1,05 \\
\hline \multirow{2}{*}{ Poste 4} & $\bar{x}$ & 0,04 & 0,00 & 1,52 & 0,01 & 0,00 & 0,00 & 18,32 & 0,00 & 0,00 & 0,92 & 0,21 & 0,00 \\
\hline & 8 & 0,09 & 0,00 & 0,66 & 0,02 & 0,00 & 0,00 & 7,66 & 0,00 & 0,00 & 0,66 & 0,12 & 0,00 \\
\hline \multirow{2}{*}{ Poste 5} & $\bar{x}$ & 0,00 & 0,00 & 0,41 & 0,00 & 0,00 & 0,01 & 2,74 & 0,00 & 0,00 & 0,26 & 0,01 & 1,17 \\
\hline & $s$ & $(1,0)$ & $(0,0)$ & $(0,34$ & $01,(11)$ & $(0,0)$ & 10,13 & 1,61 & 0,010 & $(1,1)(1)$ & 0,17 & 0,113 & 1,113 \\
\hline \multirow{2}{*}{ Poste 6} & $\bar{x}$ & 0,00 & 0,02 & 1,30 & 0,00 & 0,00 & 0,02 & 16,03 & 0,00 & 0,00 & 0,59 & 0,03 & 1,24 \\
\hline & $s$ & 0,00 & 0,05 & 0,33 & 0,00 & 0,00 & 0,05 & 4,02 & 0,00 & 0,00 & 0,29 & 0,05 & 1,62 \\
\hline \multirow{2}{*}{$\begin{array}{l}\text { Pcdra } \\
\text { Cardinal }\end{array}$} & $\bar{x}$ & 0,10 & 0,03 & 2,40 & 0,00 & 0,00 & 0,00 & 21,67 & 0,00 & 0,02 & 2,34 & 0,17 & 0,28 \\
\hline & $s$ & 0,14 & 0,08 & 1,17 & 0,00 & 0,00 & 0,00 & 5,43 & 0,00 & 0,05 & 1,41 & 0,07 & 0,32 \\
\hline \multirow{2}{*}{ Pedra Alva } & $\bar{X}$ & 0,00 & 0,00 & 0,12 & 0,00 & 0,00 & 0,00 & 12,94 & 0,00 & 0,00 & 0,01 & 0,04 & 0,00 \\
\hline & $\mathrm{s}$ & 0,00 & 0,00 & 0,13 & 0,00 & 0,00 & 0,00 & 5,59 & 0,00 & 0,00 & 0,03 & 0,06 & 0,00 \\
\hline \multirow{2}{*}{$\begin{array}{l}\text { Pedra da } \\
\text { Mangueira }\end{array}$} & $\bar{x}$ & 0,08 & 0,00 & 1,02 & 0,00 & 0,00 & 0,00 & 6,20 & 0,00 & 0,00 & 0,96 & 0,13 & 0,00 \\
\hline & $s$ & 0,15 & 0,00 & 0,49 & 0,00 & 0,00 & 0,00 & 4,80 & 0,00 & 0,00 & 0,69 & 0,17 & 0,00 \\
\hline \multirow{2}{*}{$\begin{array}{l}\text { Pedra do } \\
\text { Dentão }\end{array}$} & $\bar{x}$ & 0,00 & 0,00 & 0,00 & 0,00 & 0,00 & 0,00 & 0,66 & 0,00 & 0,00 & 0,01 & 0,00 & 0,05 \\
\hline & s & 0,00 & 0,00 & 0,00 & 0,00 & 0,00 & 0,00 & 0,44 & 0,00 & 0,00 & 0,02 & 0,00 & 0,10 \\
\hline \multirow{2}{*}{$\begin{array}{l}\text { Ilha dos } \\
\text { l'rades Leste }\end{array}$} & $\bar{X}$ & 0,00 & 0,00 & 0,17 & 0,00 & 0,00 & 0,00 & 0,00 & 0,00 & 0,00 & 0,00 & 0,00 & 0,70 \\
\hline & 5 & 0,00 & 0,00 & 0,07 & 0,00 & 0,00 & 0,00 & 0,00 & 0,00 & 0,00 & 0,00 & 0,00 & 0,97 \\
\hline \multirow{2}{*}{$\begin{array}{l}\text { Tha dos } \\
\text { Frades Sul }\end{array}$} & $\bar{x}$ & 0,03 & 0,06 & 1,50 & 0,03 & 0,00 & 0,00 & 11,19 & 0,00 & 0,00 & 0,16 & 0,04 & 0,22 \\
\hline & 8 & 0,08 & 0,12 & 0,25 & 0,08 & 0,00 & 0,00 & 2,58 & 0,00 & 0,00 & 0,07 & 0,05 & 0,34 \\
\hline \multirow{2}{*}{$\begin{array}{l}\text { Tha de } \\
\text { Maré }\end{array}$} & $\bar{X}$ & 0,00 & 0,12 & 0,76 & 0,00 & 0,00 & 0,02 & 1,68 & 0,00 & 0,00 & 0,16 & 0,00 & 0,58 \\
\hline & $\mathrm{s}$ & 0,00 & 0,29 & 0,40 & 0,00 & 0,00 & 0,03 & 1,41 & 0,00 & 0,00 & 0,22 & 0,00 & 0,66 \\
\hline & $\bar{x}$ & 0,00 & 0,00 & 0,45 & 0,00 & 0,03 & 0,05 & 6,11 & 0,00 & 0,00 & 0,08 & 0,00 & 0,19 \\
\hline Boa ViagetI & $\mathrm{s}$ & 0,00 & 0,00 & 0,38 & 0,00 & 0,07 & 0,08 & 5,02 & 0,00 & 0,00 & 0,14 & 0,00 & 0,47 \\
\hline Deri-Deri & $\bar{x}$ & 0,00 & 0,00 & 0,35 & 0,00 & 0,00 & 0,00 & 3,91 & 0,00 & 0,00 & 0,24 & 0,00 & 0,37 \\
\hline 'Cr1-1'cr1 & 8 & $0,0)$ & $0,0()$ & 0,20 & 0,00 & $(0,0)$ & 0,00 & $1, y()$ & $(0,0)$ & $(0,()(0)$ & 0,38 & $0,(0)$ & $(0,70$ \\
\hline & $\bar{x}$ & 0,00 & 0,03 & 0,36 & 0,00 & 0,00 & 0,00 & 0,30 & 0,00 & 0,00 & 0,40 & 0,00 & 0,70 \\
\hline 10 & 5 & 0,00 & 0,05 & 0,29 & 0,00 & 0,00 & 0,00 & 0,25 & 0,00 & 0,00 & 0,39 & 0,00 & 1,06 \\
\hline & $\bar{x}$ & 0,00 & 0,00 & 0,18 & 0,00 & 0,00 & 0,00 & 4,21 & 0,00 & 0,00 & 0,05 & 0,02 & 0,52 \\
\hline & $\mathrm{s}$ & 0,00 & 0,00 & 0,32 & 0,00 & 0,00 & 0,00 & 2,43 & 0,00 & 0,00 & 0,12 & 0,05 & 0,61 \\
\hline
\end{tabular}

$-10-$ 
Cruz, Kikuchi e L eão

Revista de G estão C osteira Integrada / Journal of Integrated C oastal Z one M anagement 9(3):3-23 (2009)

Tabela 3: Matriz de composição da comunidade (presença e ausência). Destaca-se em cinza escuro as espécies que ocorreram com exclusividade nos recifes externos ou internos.

Table 3: Matrix of coral composition (presenœ and absenœ). In dark gray species that ocaurred ex clusively in the ex ternal or internal reefs.

\begin{tabular}{|c|c|c|c|c|c|c|c|c|c|c|c|c|c|c|c|c|c|}
\hline & & 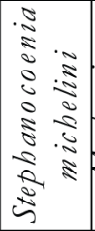 & 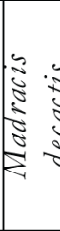 & 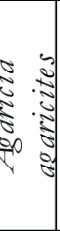 & 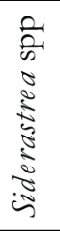 & $\begin{array}{rl}3 \\
3 & 2 \\
2 & 0 \\
0 & 0 \\
0 & 0 \\
0 & 0 \\
0 & 0 \\
0\end{array}$ & 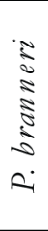 & 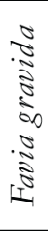 & 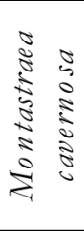 & 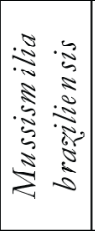 & 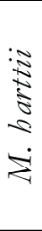 & 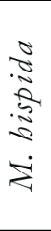 & 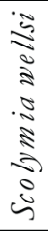 & 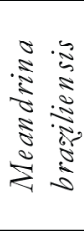 & 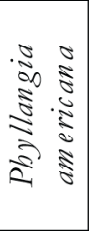 & 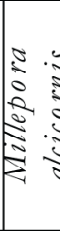 & 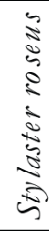 \\
\hline \multirow{8}{*}{ 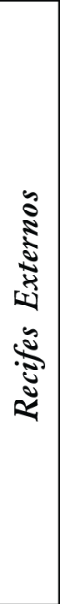 } & \begin{tabular}{|l} 
Caramuana \\
s Norte \\
\end{tabular} & & & 1 & 1 & 1 & 1 & & 1 & 1 & 1 & 1 & & & & 1 & \\
\hline & $\begin{array}{l}\text { Caramuana } \\
\text { s Leste- } \\
\text { sueste } \\
\end{array}$ & & & 1 & 1 & 1 & 1 & 1 & 1 & 1 & 1 & 1 & & & & 1 & 1 \\
\hline & \begin{tabular}{|l} 
Caramuana \\
s Sul \\
\end{tabular} & & & 1 & 1 & 1 & 1 & 1 & 1 & 1 & 1 & 1 & & & & 1 & 1 \\
\hline & Pinaúnas 1 & & & 1 & 1 & & 1 & & 1 & 1 & 1 & 1 & & & & & \\
\hline & Pinaúnas 2 & & & & 1 & & 1 & & & 1 & & & & & & & \\
\hline & Pinaúnas 3 & & & 1 & 1 & & 1 & & 1 & 1 & 1 & 1 & & & & & \\
\hline & Pinaúnas 4 & & & & 1 & & 1 & & & 1 & & 1 & & & & & \\
\hline & Pinaúnas 5 & & & & 1 & & 1 & & & 1 & & 1 & & & & & \\
\hline \multirow{15}{*}{ 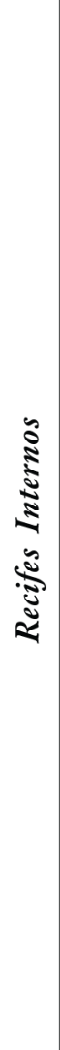 } & Poste 1 & 1 & 1 & & 1 & & & & 1 & & & 1 & 1 & & & 1 & \\
\hline & Poste 4 & 1 & 1 & & 1 & 1 & & & 1 & & & 1 & 1 & & & 1 & \\
\hline & Poste 5 & 1 & & 1 & 1 & & & 1 & 1 & & & 1 & 1 & & & 1 & \\
\hline & Poste 6 & 1 & & 1 & 1 & & & 1 & 1 & & & 1 & 1 & & 1 & 1 & \\
\hline & $\begin{array}{l}\text { Pedra } \\
\text { Cardinal }\end{array}$ & 1 & 1 & 1 & 1 & & & & 1 & & 1 & 1 & 1 & & 1 & 1 & \\
\hline & Prdra Nlva & 1 & 1 & & 1 & & & & 1 & & & 1 & 1 & & & & \\
\hline & $\begin{array}{l}\text { Pedra da } \\
\text { Mangueira }\end{array}$ & 1 & 1 & & 1 & & & & 1 & & & 1 & 1 & & 1 & & \\
\hline & $\begin{array}{l}\text { Pedra do } \\
\text { Dentão }\end{array}$ & 1 & & & 1 & & & & 1 & & & 1 & 1 & & & 1 & \\
\hline & $\begin{array}{l}\text { Ilha dos } \\
\text { Frades } \\
\text { Leste } \\
\end{array}$ & & & & 1 & 1 & & & 1 & & & & & & & 1 & \\
\hline & $\begin{array}{l}\text { Ilha dos } \\
\text { Frades Sul }\end{array}$ & 1 & 1 & 1 & 1 & 1 & & 1 & 1 & & & 1 & 1 & & & 1 & \\
\hline & $\begin{array}{l}\text { Ilha de } \\
\text { Maré }\end{array}$ & 1 & & 1 & 1 & 1 & & 1 & 1 & & & 1 & 1 & 1 & & 1 & \\
\hline & Boa Viagem & & & 1 & 1 & & 1 & 1 & 1 & & 1 & 1 & & & & 1 & \\
\hline & Peri-Peri & & & & 1 & & & 1 & 1 & & & 1 & 1 & & & 1 & \\
\hline & Tubarão & & & 1 & 1 & 1 & & 1 & 1 & & & 1 & 1 & & 1 & 1 & \\
\hline & Inema & 1 & & & 1 & 1 & & & 1 & & & 1 & 1 & & & 1 & \\
\hline
\end{tabular}




\section{Estrutura da Comunidade em Grupos}

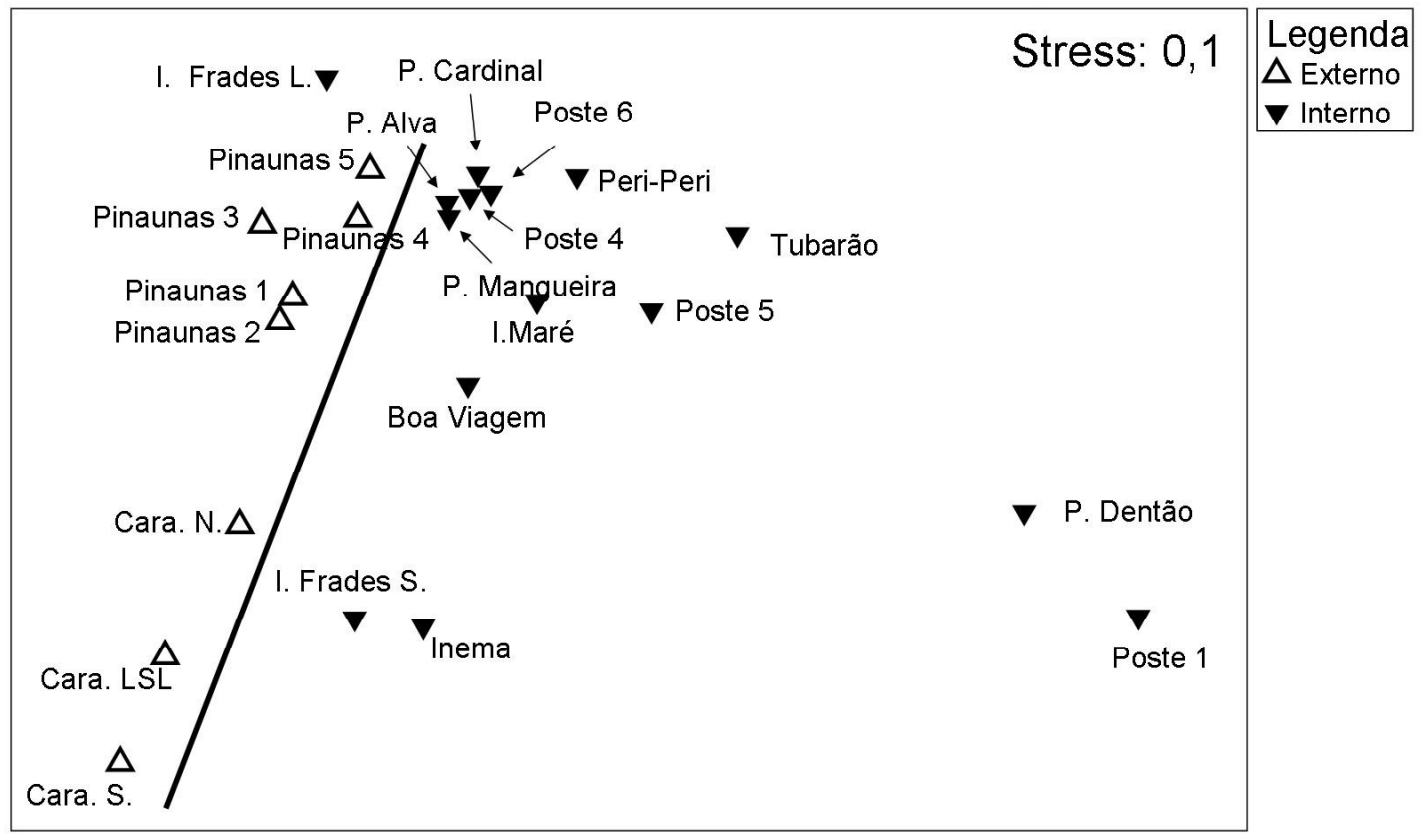

Figura 2: Gráfico de Escalonamento Multidimensional (Multi-D imensional Scaling MDS) para a estrutura da comunidade macrobentônica.

Figure 2: G raph Multi-D imensional Scaling (M D S) for macrobenthonic community structure

\section{Estrutura de Comunidade de Coral}

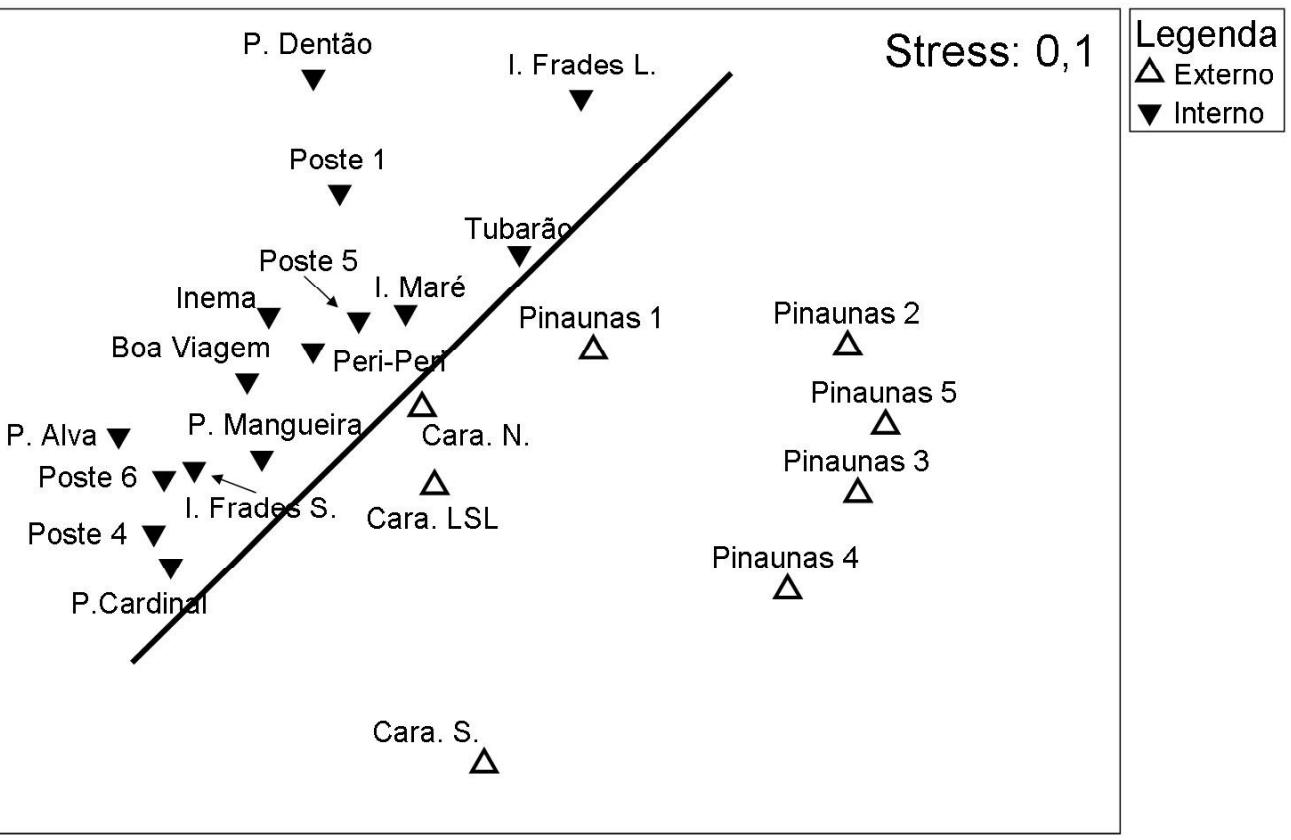

Figura 3: Gráfico Escalonamento Multidimensional (M ulti-D imensional Scaling MDS) para estrutura da comunidade de coral.

Figure 3: G raph M ulti-D imensional Scaling (M D S) for the coral community structure. 
Cruz, Kikuchi e L eão

R evista de G estão C osteira Integrada / Journal of Integrated C oastal Z one M anagement 9(3):3-23 (2009)

\section{Recifes externos}

Os recifes externos assemelham-se entre si, de acordo com a análise SIMPER, em 55,5\% em relação à estrutura da comunidade macrobentônica. As principais características em comum foram a distribuição e a abundância das algas filamentosas, das algas calcárias incrustantes e das algas calcárias articuladas. Entretanto, em relação à estrutura da comunidade dos corais, estes recifes assemelham-se em apenas $22 \%$. Esta semelhança está caracterizada pela distribuição e abundância dos corais Mussismilia braziliensis, M. hispida, Siderastrea spp e Porites branneri. Porem, com relação à composição da comunidade dos corais, esses recifes externos assemelham-se em $72 \%$. Esta semelhança é apontada pela ocorrência de
Sideratrea spp, M ussimilia braziliensis, M . hispida e Porites branneri (Tabela 4).

\section{Recifes internos}

De acordo com a análise SIMPER, a semelhança destes recifes entre si, quanto à estrutura da comunidade macrobentônica, é de 46,3\%, caracterizada pela distribuição e abundância das algas filamentosas, acrescida das esponjas e dos corais. Para a estrutura da comunidade dos corais, a semelhança entre estes recifes é de 40\% caracterizada pela abundância e distribuição dos corais M. cavernosa, Sideratrea spp e do hidrocoral M illepora alciornis. E em relação à composição da comunidade dos corais, os recifes internos se assemelham em $72 \%$. Contribuíram

Tabela 4: Tabela de similaridade dos recifes externos. X. abund (\%) - média da abundância em \%, X. Sim (\%) - média da similaridade em \%, s Sim - desvio padrão da similaridade e Contrib \% - a categoria contribui para o valor do Índice de Similaridade.

Table 4: Similarity table of outside reefs. $\boldsymbol{X}$. abund (\%) - abundanoe average in \%, $\boldsymbol{X}$. Sim (\%) - similarity average in \%, s Sim - similarity standard deviation e Contrib\% - ontribution to Similarity index.

\begin{tabular}{|c|c|c|c|c|c|c|}
\hline Grupo & $\begin{array}{c}\text { Índice de } \\
\text { similaridade }\end{array}$ & Organismo & $\begin{array}{c}\mathrm{X} \\
\text { Abund } \\
(\%)\end{array}$ & $\begin{array}{l}X \\
\text { Sim } \\
(\%)\end{array}$ & s Sim & Contrib $\%$ \\
\hline \multirow{3}{*}{$\begin{array}{c}\text { Estrutura de Comunidade } \\
\text { Macrobentônica }\end{array}$} & \multirow{3}{*}{$5.5,5$} & Alga filamentosa & 41,0 & 29,7 & 1,32 & 53,5 \\
\hline & & $\begin{array}{l}\text { Alga calcária } \\
\text { incrustante }\end{array}$ & 18,6 & 10,7 & 1,12 & 19,2 \\
\hline & & $\begin{array}{l}\text { Alga calcária } \\
\text { articulada }\end{array}$ & 17,7 & 9,79 & 1,10 & 17,6 \\
\hline \multirow{4}{*}{$\begin{array}{c}\text { Estrutura de Comunidade de } \\
\text { Coral }\end{array}$} & \multirow{4}{*}{23,7} & $\begin{array}{l}\text { Mussismilia } \\
\text { braziliensis }\end{array}$ & 0,13 & 8,19 & 0,76 & 34,6 \\
\hline & & Siderastrea $\mathrm{spp}$ & 1,58 & 5,69 & 0,98 & 24,0 \\
\hline & & $\begin{array}{l}\text { Mussismilia } \\
\text { hispida }\end{array}$ & 0,78 & 5,41 & 0,69 & 22,8 \\
\hline & & Porites branneri & 0,06 & 2,20 & 0,70 & 9,29 \\
\hline \multirow{6}{*}{$\begin{array}{c}\text { Composição da Comunidade } \\
\text { de Coral }\end{array}$} & \multirow{6}{*}{72,2} & Siderastrea spp & 100 & 15,6 & 3,02 & 21,6 \\
\hline & & $\begin{array}{l}\text { Mussismilia } \\
\text { braziliensis }\end{array}$ & 100 & 15,6 & 3,02 & 21,6 \\
\hline & & Porites branneri & 100 & 15,6 & 3,02 & 21,6 \\
\hline & & $\begin{array}{l}\text { Mussismilia } \\
\text { hispida }\end{array}$ & 88,0 & 10,5 & 1,50 & 14,6 \\
\hline & & $\begin{array}{l}\text { Montastraea } \\
\text { cavernosa }\end{array}$ & 63,0 & 4,03 & 0,72 & 5,6 \\
\hline & & $\begin{array}{l}\text { Agaricia } \\
\text { agaricites }\end{array}$ & 63,0 & 4,03 & 0,72 & 5,6 \\
\hline
\end{tabular}


para esta semelhança as espécies Sideratrea spp, M ontastraea cavernosa, M ussismilia hispida, Scolymia wellsi e M illepora alcioornis (Tabela 5).

\section{Diferença entre os recifes}

A diferença entre os dois grupos de recifes quanto à estrutura da comunidade macrobentônica é de 59,9\%, segundo a análise SIMPER. Esta diferença está caracterizada pela abundância e distribuição das algas filamentosas, das algas coralináceas, das algas calcárias articuladas e das algas frondosas, assim como dos E pizoanthus, das esponjas, dos corais e das cianobactérias. Em relação à estrutura da comunidade dos corais, estes recifes diferenciam-se em $84 \%$. Esta diferença baseia-se na distribuição e na abundância das espécies Montastraea cavernosa, Sideratrea spp, Mussismilia hispida e Millepora alcicornis (Tabela 6). E esses dois grupos se distinguem quanto à composição da comunidade dos corais em 55\%. Eles diferem principalmente pela maneira como se distribuem as espécies M ussismilia braziliensis, Porites branneri, Soolymia wellsi, Stephanocoenia michelini (Milne Edwards e Haime, 1795), M ussismilia harttii, A garicia agaricites, M ontastraea cavernosa, Favia gravida, Porites astreoides, M adracis decatis e Millepora alcioornis (Tabela 6).

\section{Discussão:}

\section{Recifes Externos}

A composição da comunidade dos corais foi o parâmetro que melhor agrupou os recifes externos, apresentando uma similaridade de $72,16 \%$. Este valor indica que as espécies que ocorrem nestes recifes são recorrentes. Entretanto, o MDS para a composição da comunidade dos corais (Figura 4) mostra que as estações do grupo das Caramuanas e dos recifes das

Tabela 5: Tabela de similaridade dos recifes internos. X. abund (\%) - média da abundância em \%, X. Sim (\%) - média da similaridade em \%, s Sim - desvio padrão da similaridade e Contrib\% - a categoria contribui para o valor do Índice de Similaridade.

Table 5: Similarity table of outside reefs. $\boldsymbol{X}$. abund (\%) - abundanœ average in \%, $\boldsymbol{X}$. Sim (\%) - similarity average in $\%, \boldsymbol{s}$ Sim - similarity standard deviation e Contrib\% - contribution to Similarity index .

\begin{tabular}{|c|c|c|c|c|c|c|}
\hline Grupo & $\begin{array}{l}\text { Índice de } \\
\text { similaridade }\end{array}$ & Organismo & $\begin{array}{c}\mathrm{X} \\
\text { Abund } \\
(\%)\end{array}$ & $\begin{array}{c}\mathrm{X} \\
\mathrm{Sim} \\
(\%)\end{array}$ & s Sim & Contrib $\%$ \\
\hline \multirow{3}{*}{$\begin{array}{c}\text { Extrutura de Comunidade } \\
\text { Macrobentônica }\end{array}$} & \multirow{3}{*}{46,3} & Alga filamentosa & 33,6 & 29,7 & 1,64 & 64,2 \\
\hline & & Esponja & 8,80 & 7,51 & 1,63 & 16,2 \\
\hline & & Coral & 8,86 & 5,18 & 1,10 & 11,8 \\
\hline \multirow{3}{*}{$\begin{array}{l}\text { Extrutura de Comunidade } \\
\text { de Coral }\end{array}$} & \multirow{3}{*}{40,9} & $\begin{array}{l}\text { Montastraea } \\
\text { cavernosa }\end{array}$ & 7,19 & 30,6 & 1,26 & 74,8 \\
\hline & & $\begin{array}{l}\text { Millepora } \\
\text { alcicornis }\end{array}$ & 0,44 & 5,14 & 0,57 & 12,6 \\
\hline & & Siderastrea spp & 0,71 & 3,71 & 1,08 & 9,08 \\
\hline \multirow{7}{*}{$\begin{array}{c}\text { Composição da Comunidade } \\
\text { de Coral }\end{array}$} & \multirow{7}{*}{72,7} & Siderastrea spp & 100 & 14,0 & 6,15 & 18,5 \\
\hline & & $\begin{array}{l}\text { Montastraea } \\
\text { cavernosa }\end{array}$ & 100 & 14,0 & 6,15 & 18,5 \\
\hline & & $\begin{array}{l}\text { Mussismilia } \\
\text { bispida }\end{array}$ & 93,0 & 11,2 & 2,38 & 15,9 \\
\hline & & $\begin{array}{l}\text { Millepora } \\
\text { alcicornis }\end{array}$ & 87,0 & 9,8 & 1,52 & 13,5 \\
\hline & & Scolymia wellsi & 87,0 & 9,62 & 1,63 & 13,2 \\
\hline & & $\begin{array}{l}\text { Stepbanocoenia } \\
\text { michelini }\end{array}$ & 73,0 & 6,72 & 1,02 & 9,25 \\
\hline & & Favia gravida & 47,0 & 2,40 & 0,49 & 3,30 \\
\hline
\end{tabular}


Cruz, Kikuchi e L eão

Revista de G estão C osteira Integrada / Journal of Integrated C oastal Z one M anagement 9(3):3-23 (2009)

Tabela 6: Tabela de dissimilaridades entre os recifes externos e internos. X. abund (\%) - média da abundância em \%, X. Diss (\%) - média da dissimilaridade em \%, $\mathbf{s}$ Diss - desvio padrão da similaridade e Contrib\% - a categoria contribui para o valor do Índice de Dissimilaridade.

Table 6: D issimilarity table betwen outside and inside refs. $\boldsymbol{X}$. abund (\%) - abundance average in \%, $\boldsymbol{X}$. Diss (\%) dissimilarity average in \%, $\boldsymbol{s}$ Diss - dissimilarity standard deviation e Contrib\% - D issimilarity index contribution.

\begin{tabular}{|c|c|c|c|c|c|c|c|}
\hline Grupo & $\begin{array}{c}\text { Índice de } \\
\text { dissimilaridade }\end{array}$ & Organismo & $\begin{array}{r}\text { X. Abu } \\
\text { Recifes } \\
\text { Externos }\end{array}$ & $\begin{array}{l}\text { nd }(\%) \\
\text { Recifes } \\
\text { Internos }\end{array}$ & $\begin{array}{c}\mathrm{X} \\
\text { Diss } \\
(\% / 0) \\
\end{array}$ & $\begin{array}{c}\text { S } \\
\text { Diss }\end{array}$ & $\begin{array}{c}\text { Contrib } \\
(\%)\end{array}$ \\
\hline \multirow{8}{*}{$\begin{array}{l}\text { Extrutura de } \\
\text { Comunidade } \\
\text { Macrobentônica }\end{array}$} & \multirow{8}{*}{60,0} & Alga filamentosa & 41,0 & 33,6 & 13,7 & 1,42 & 22,8 \\
\hline & & $\begin{array}{l}\text { Alga calcária } \\
\text { incrustante }\end{array}$ & 18,6 & 0,33 & 10,8 & 1,26 & 17,9 \\
\hline & & $\begin{array}{l}\text { Alga calcária } \\
\text { articulada }\end{array}$ & 17,6 & 5,00 & 10,1 & 1,29 & 16,85 \\
\hline & & Epizoanthus & 0,01 & 10,9 & 5,88 & 0,44 & 9,81 \\
\hline & & Esponja & 2,50 & 8,80 & 4,59 & 1,19 & 7,65 \\
\hline & & Alga frondosa & 4,41 & 5,41 & 4,46 & 0,82 & 7,44 \\
\hline & & Coral & 3,38 & 8,86 & 4,36 & 1,16 & 7,27 \\
\hline & & Cianobactéria & 5,33 & 1,86 & 3,41 & 0,81 & 5,69 \\
\hline \multirow{4}{*}{$\begin{array}{c}\text { Extrutura de } \\
\text { Comunidade de } \\
\text { Coral }\end{array}$} & \multirow{4}{*}{84,6} & $\begin{array}{c}\text { Montastraea } \\
\text { cavernosa }\end{array}$ & 0,48 & 19,0 & 49,8 & 1,7 & 58,8 \\
\hline & & Siderastrea spp & 1,58 & 71,0 & 13,0 & 0,74 & 15,4 \\
\hline & & Millepora alcicornis & 0,23 & 44,0 & 8,96 & 0,7 & 10,6 \\
\hline & & Mussismilia hispida & 0,78 & 12,0 & 8,05 & 0,85 & 9,52 \\
\hline \multirow{11}{*}{$\begin{array}{l}\text { Composição da } \\
\text { Comunidade de } \\
\text { Coral }\end{array}$} & \multirow{11}{*}{55,6} & $\begin{array}{c}\text { Mussismilia } \\
\text { braziliensis }\end{array}$ & 100 & 0,00 & 7,27 & 3,86 & 13,0 \\
\hline & & Porites branneri & 100 & 7,00 & 6,81 & 2,62 & 12,3 \\
\hline & & Scolymia wellsi & 0,00 & 87,0 & 6,15 & 2,13 & 11,1 \\
\hline & & $\begin{array}{c}\text { Stephanocoenia } \\
\text { michelini }\end{array}$ & 0,00 & 73,0 & 5,18 & 1,51 & 9,32 \\
\hline & & Millepora alcicornis & 38,0 & 87,0 & 4,71 & 1,13 & 8,47 \\
\hline & & Mussismilia harttii & 63,0 & 13,0 & 3,78 & 1,16 & 6,81 \\
\hline & & Agaricia agaricites & 63,0 & 47,0 & 3,59 & 0,99 & 6,45 \\
\hline & & $\begin{array}{c}\text { Mo ntastraea } \\
\text { caverno sa }\end{array}$ & 63,0 & 100 & 3,42 & 0,75 & 6,15 \\
\hline & & Favia gravida & 25,0 & 17,0 & 3,32 & 0,93 & 5,97 \\
\hline & & Porites astreoides & 38,0 & 40,0 & 3,31 & 0,89 & 5,95 \\
\hline & & Madracis decatis & 0,00 & 40,0 & 2,81 & 0,78 & 5,06 \\
\hline
\end{tabular}

Pinaunas não se misturam. Isso se deve à existência de uma espécie de coral, Favia gravida e duas de hidrocorais Millepora alciornis e Stylaster roseus, que ocorrem nos recifes das Caramuanas, as quais não foram encontradas nos recifes das Pinaunas (Tabela 3).

Embora a composição das espécies de corais tenha apresentado um alto grau de semelhança entre os recifes, a estrutura da comunidade mostrou uma baixa similaridade, de apenas $23,68 \%$. Isso sugere que embora as espécies sejam recorrentes, elas estão distribuídas com abundâncias muito distintas. O MDS para a estrutura da comunidade dos corais (Figura 3) mostra as estações mais separadas, o que é esperado com um índice de similaridade baixo. Contudo, mais uma vez as estações dos recifes das Caramuanas não 
Cruz, Kikuchi e L eão

R evista de G estão C osteira Integrada / Journal of Integrated C oastal Z one M anagement 9(3):3-23 (2009)

Figura 4: Gráfico Escalonamento Multidimensional (Multi-D imensional Scaling MDS) para composição da comunidade de coral.

Figure 4: G raph Multi-D imensional Scaling (MD S) for the coral ommunity omposition.

Composição da Comunidade de Coral

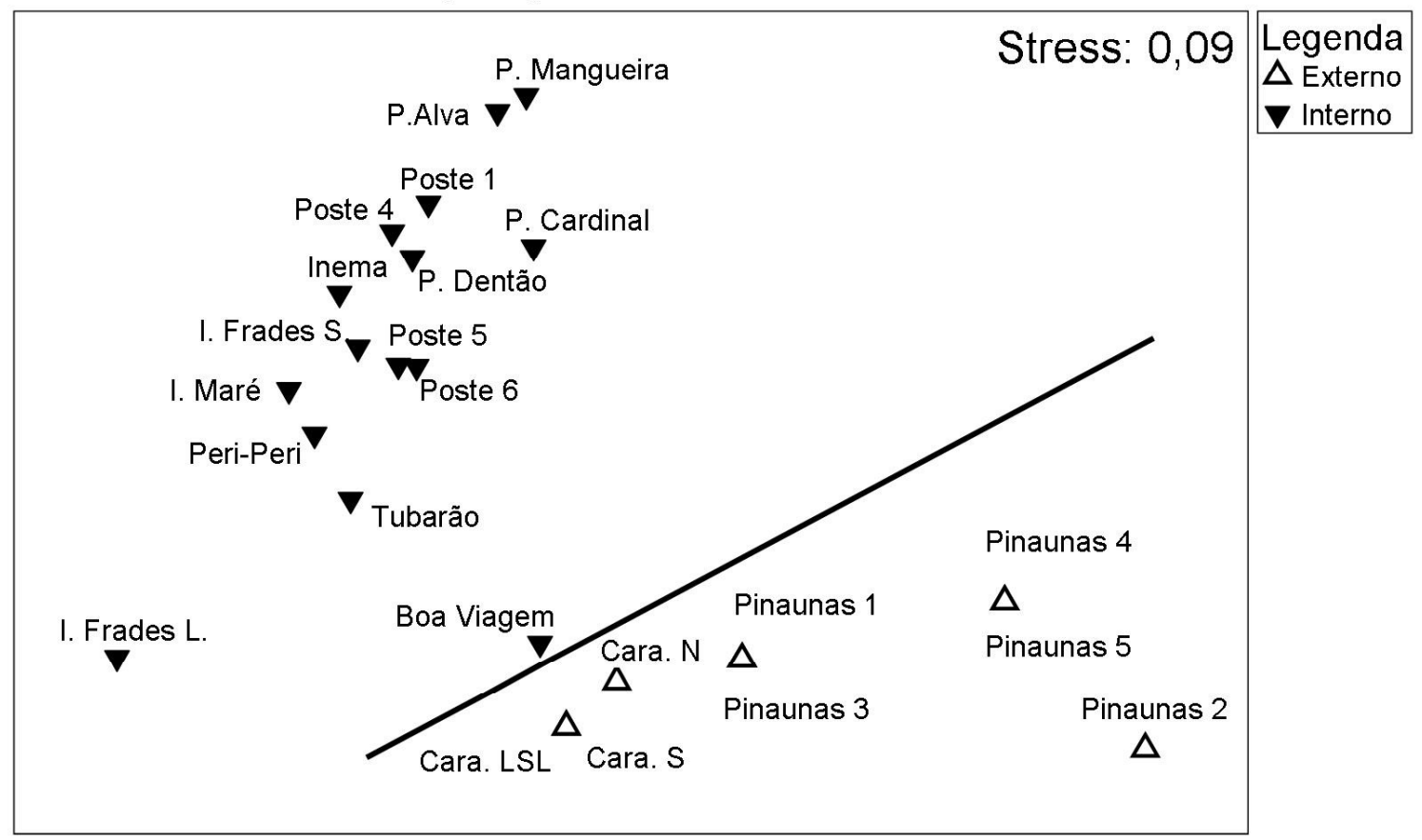

se misturam com as estações do recife das Pinaunas. Esta separação justifica-se pela ausência em Pinaunas das espécies A gaaricia agaricites, Porites astreoides, Favia gravida e Mussismilia harttii e pela menor abundância e ocorrência das espécies do complexo Siderastrea, e das espécies Montastraea cavernosa, Mussismilia hispida e Millepora alciornis (Tabela 2). Dentre todas as estações externas destaca-se, no gráfico, a de Caramuanas Sul, que embora apresente as mesmas espécies que em Caramuanas Leste-Sueste, encontra-se mais isolada devido a maior abundância dos corais do complexo Siderastrea que ocorrem em toda a BTS (Tabela 2, Figura 3).

A estrutura da comunidade macrobentônica, por sua vez, apresenta um valor intermediário no índice de similaridade, 55,52\%. A análise SIMPER caracteriza os recifes externos pela alta abundância de alga filamentosa, alga calcária incrustante e alga calcária articulada (Tabela 4). Contudo, no gráfico MDS para a estrutura dessa comunidade macrobentônica (Figura 2) é possível observar que as estações do recife das Pinaunas e das Caramuanas além de não se misturarem, elas apresentam, também, uma tendência a agruparem de maneira mais evidente. Entre as divergências, destacam-se a cobertura de coral, as algas filamentosas, as cianobactérias, as algas calcárias incrustantes e as algas calcárias articuladas (Tabela 1). Os recifes das Caramuanas apresentam uma cobertura de coral vivo variando entre 6,5 e $10,4 \%$, de alga calcária incrustante entre 32,2 e $41,6 \%$ e de alga calcária articulada entre 20,5 e 44,4\%, enquanto que nos recifes das Pinaunas a cobertura de coral vivo varia entre 0,2 a 1,12\%, de alga calcária incrustante entre 2,5 e $13,2 \%$ e de alga calcária articulada entre 0,7 e $16,9 \%$. Por sua vez os recifes das Pinaunas apresentam uma maior abundância de alga filamentosa, que varia entre 55,3 a $64,3 \%$ e de cianobactéria entre 0,12 a $21,1 \%$, enquanto que os recifes das Caramuanas a cobertura de alga filamentosa varia entre 5,4 e 22,7\% e de cianobactéria 
entre zero e $0,08 \%$.

Com base nas análises SIMPER os recifes externos caracterizam-se, principalmente, pela ocorrência e abundância de alga filamentosa, alga calcária incrustante, alga calcária articulada, e dos corais M ussismilia braziliensis, o complexo Siderastrea, Mussismilia hispida e Porites branneri. Entretanto estes recifes estão separados em dois grupos. A abundância média de 41\% $\pm 23,2$, de alga filamentosa (Tabela 4) nos recifes das Pinaunas não corresponde à quantidade das mesmas nos recifes das Caramuanas. Além disso, estes recifes apresentam uma maior riqueza de espécies e uma maior cobertura de coral que os recifes das Pinaunas. Estes são dois importantes critérios de integridade recifal (Jamenson et al., 1998; Jamenson et al., 2001; McField \& Kramer, 2007), a qual indica uma degradação maior nos recifes das Pinaunas. O fato do recife das Pinaunas localizarse mais próximo da costa da ilha de Itaparica (menos que $1 \mathrm{~km}$ ) e da região mais habitada desta ilha, o torna mais susceptível à degradação pela ação humana.

\section{Recifes Internos}

Para os recifes internos a composição da comunidade dos corais foi, também, o parâmetro que melhor os agrupou. O índice de similaridade foi de $72,69 \%$. As espécies do complexo Siderastrea e a espécie M ontastraea cavernosa ocorreram em todas as estações, Mussismilia hispida ocorreu em 93\% das estações, enquanto que Millepora alcicornis e Scolymia wellsi em 87\% e Stephanocenia michelini em 73\% (Tabela 5). O MDS para a composição da comunidade dos corais mostra que dois dos recifes internos, Boa Viagem e Ilha dos Frades Leste, estão um pouco separados dos demais (Figura 4). O que provavelmente segrega o recife Ilha dos Frades Leste é a ausência de três espécies muito comuns nos demais recifes internos. Esta foi a única estação interna onde não ocorreu M ussismilia hispida. Além desta espécie, estiveram ausente Scolymia wellsi e Stephanocoenia michelini que segundo a análise SIMPER correspondem juntas a $22,5 \%$ da similaridade dos recifes internos (Tabela 5). Esta duas espécies não ocorrem em lugares rasos. A estação de Ilha dos Frades Leste foi a mais rasa de todas no interior da BTS, com apenas dois metros de profundidade. A estação de Boa Viagem é a única estação interna onde ocorreu a espécie Porites branneri.

Embora a estrutura da comunidade dos corais tenha apresentado o menor índice de similaridade para os recifes internos, 40,86\% (Tabela 5), este índice não foi tão baixo quanto para os recifes externos. As três espécies indicadas pela analise SIMPER como responsáveis por esta similaridade são as mais abundantes nos recifes internos. M ontastraea cavernosa é a espécie dominante e corresponde sozinha a 74,8\% dessa semelhança (Tabela 5). Já as espécies do complexo Siderastrea e Millepora alcicornis são espécies muito comuns e juntas representam 21,66\% desta similaridade.

A estrutura da comunidade dos organismos macrobentônicos mostrou uma similaridade de 46,3\% para os recifes internos (Tabela 5). Os principais responsáveis por esta semelhança foram a abundancia e a distribuição de alga filamentosa, de esponja e de coral (Tabela 5). A análise MDS para a estrutura da comunidade desses organismos (Figura 2) mostra dois grupos distanciados dos recifes internos. As estações Pedra do Dentão e Poste 1 apresentam abundâncias destoantes de Epizoanthus sp, das demais estações (Tabela 1). Por sua vez, as estações Ilha dos Frades Sul e Inema apresentam uma alta abundância, acima de $20 \%$, de alga calcária articulada, o que, entretanto, as aproxima dos recifes externos, a exemplo das estações dos recifes das Caramuanas (Tabela 1).

De acordo com as análises SIMPER, os recifes internos são caracterizados pela dominância de alga filamentosa com uma média de 33,6\% $\pm 17,8$, além da alta abundância de esponjas e corais, respectivamente $8,9 \% \pm 6,2$ e $8,8 \% \pm 8,1$ (Tabela 5). As espécies de corais mais comuns são Montastraea cavernosa, o complexo Siderastrea, M ussismilia hispida, Millepora alcicornis, Scolymia wellsi e Stephanocoenia michelini estando presentes em mais de $70 \%$ das estações (Tabela 5). Destas, M ontastraea cavernosa é a espécie dominante com uma média de cobertura de $7,2 \% \pm 7,1$. Apresentam, ainda, uma alta abundancia das espécies do complexo Siderastrea e Millepora alcicornis (Tabela 5).

\section{Diferenças entre os recifes}

A estrutura da comunidade dos corais apresenta a maior diferença entre os recifes externos e internos, com um índice de dissimilaridade de 84,57\% (Tabela 


\section{Cruz, Kikuchi e L eão \\ Revista de G estão C osteira Integrada / Journal of Integrated C oastal Z one M anagement 9(3):3-23 (2009)}

6). Este resultado mostra que existe uma diferença marcante não só na composição mas, também, na abundancia das espécies de corais entre os dois grupos de recifes.

A análise SIMPER indica que os recifes externos diferenciam-se dos internos pela maior abundância das espécies do complexo Siderastrea e Mussismilia hispida (Tabela 6). Entretanto, os valores desta análise não correspondem às abundancias dessas espécies nos recifes das Pinaunas. Apenas as estações dos recifes das Caramuanas possuem cobertura destas espécies maior ou igual que as médias das coberturas das espécies dos recifes internos (Tabela 2). Estas espécies e suas abundâncias diferenciam apenas os recifes das Caramuanas dos recifes internos.

Os recifes internos diferenciam-se dos externos quanto à estrutura da comunidade de coral pela maior ocorrência e abundância das espécies Montastraea cavernosa e Millepora alcicornis (Tabela 6). Destaca-se nesse resultado o fato da espécie do hidrocoral $\mathrm{M}$. alcicornis, que é encontrada com maior abundância em áreas de maior hidrodinâmica (Hubbard, 1997; Reigl \& Piller, 1999), ser mais abundante nos recifes internos. Segundo Araújo (1984), na década de oitenta do século passado, esta espécie cobria a frente recifal dos recifes das Pinaunas e era abundante nos recifes das Caramuanas, na faixa de profundidade entre dois e quatro metros. Durante esta pesquisa esta espécie não foi encontrada nos recifes das Pinaunas e em Caramuanas sua abundância foi baixa (Tabela 2).

Uma possível explicação para as mudanças na distribuição e na abundância de Millepora alcicornis é a pesca predatória com explosivos e redes de espera. Por ser ramificado, esse hidrocoral prende as redes de espera postas sobre o recife, provocando a quebra das colônias. E essas redes, quando presas nos ramos de M illepora, são cortadas pelos pescadores e deixadas sobre o recife, amplificando seu impacto. A pesca com bomba, a depender de sua intensidade, promove impactos que levam décadas para serem recuperados, quando não geram danos irreversíveis nos recifes (Riegl e Luke 1998; Riegl, 2001). Essa modificação foi tão intensa que este organismo de ambiente de alta energia apresentou uma abundância menor nos recifes externos que nos internos sendo responsável por $10,6 \%$ da diferença entre estes dois grupos para a estrutura da comunidade dos corais (Tabela 6).
O índice de dissimilaridade para a composição da comunidade dos corais corresponde a 55,56\% (Tabela $6)$. Os recifes externos apresentam duas espécies que não ocorrem nos internos, Mussismilia braziliensis e Stylaster roseus (Tabela 3). E a espécie Porites branneri ocorre em todas as estações dos recifes externos e em apenas uma estação dos internos, a de Boa Viagem, que se localiza bem próxima dos recifes externos, na entrada da BTS (Tabela 3). A espécie M ussismilia harttii ocorre em $63 \%$ dos recifes externos e nos internos apenas em Boa Viagem e Pedra Cardinal (Tabela 3). Este coral possui uma grande variedade morfológica com duas formas muito comuns na BTS, a laxa e a conferta (Laborel, 1969b; Verrill, 1903). A forma laxa pode ser identificada através da técnica de videotransecto ou por visualização em campo. Já a forma conferta assemelha-se muito à espécie M ussismilia hispida, podendo ser diferenciada, apenas, observando a porção inferior da colônia (Laborel, 1969b; Verrill, 1903). Para verificar a distribuição dessa população de Mussismilia harttii se faz necessário a coleta de espécimes, o que implica em método destrutivo, não adequado para um trabalho com fins de conservação. Por sua vez nos recifes internos ocorreram cinco espécies que não foram encontradas nos recifes externos Stephanocoenia michelini, Madracis decatis, Scolymia wellsi, M eandrina braziliensis e Phyllangia americana (Tabela 3).

A estrutura de comunidade dos organismos macrobentônicos apresentou um índice de dissimilaridade de 59,99\% (Tabela 6). Segundo a análise SIMPER, os recifes externos diferenciam-se dos internos pela maior abundancia de alga filamentosa, alga calcária incrustante, alga calcária articulada e cianobactéria (Tabela 6). Entretanto as médias de alga filamentosa, 41\% $\pm 23,2$ e de cianobactéria, 5,3\% $\pm 7,8$ não correspondem aos valores dos recifes das Caramuanas para estas classes de organismos (Tabela 1 e 6 ). Por conseqüência, os recifes externos diferenciam-se dos internos pela maior abundancia de alga calcária incrustante e alga calcária articulada. Os recifes das Pinaunas diferenciam-se dos recifes internos e dos recifes das Caramuanas pela maior abundância de alga filamentosa e cianobactéria.

Os recifes internos diferenciam-se dos externos, segundo a análise SIMPER, pela maior abundância 
de E pizoanthus sp, esponjas, algas frondosas e corais (Tabela 6). Contudo, a média de abundância de E pizoanthus sp de 10,93\% $\pm 26,3$ foi elevada devido aos valores das estações Poste 1 e Pedra do Dentão com $82,7 \%$ e 67,5\%, respectivamente (Tabelas 1 e 6 ). Desta maneira este organismo não representa os recifes internos e como conseqüência o mesmo não deve ser usado para diferenciar os dois grupos de recifes.

\section{CONCLUSÃO}

Os recifes externos da BTS diferenciam-se dos internos pela alta cobertura de alga calcária incrustante $(18,6 \% \pm 10,6)$ e de alga calcária articulada $(17,6 \%$ $\pm 9,8)$. Possuem com exclusividade as espécies Mussismilia braziliensis e Stylaster roseus e uma maior distribuição de Porites branneri, A garicia agaricites e M ussismilia harttii forma lax a. Por outro lado, os recifes internos caracterizam-se pela maior quantidade de esponjas $(8,8 \% \pm 7,5)$ e cobertura de coral $(8,9 \% \pm 5,5)$. Apresentam com exclusividade os corais Scolymia wellsi, Stephanocoenia michelini e Madracis decactis e uma maior abundância e distribuição espacial de Montastraea cavernosa, Favia gravida, Porites astreoides e Millepora alcioornis.

A estrutura da comunidade dos organismos macrobentônicos e a estrutura da comunidade dos corais são diferentes nos recifes externos e internos, da mesma maneira que os padrões de hidrodinâmica e os impactos causados pela poluição de origem industrial. O fato das espécies M ussismilia braziliensis e Stylaster roseus ocorrerem apenas nos recifes externos e as espécies Soolymia wellsi, Stephanocoenia michelini, Madracis decactis e Meandrina braziliensis ocorrerem, apenas, nos recifes internos justificam a criação de Zonas de Preservação da Vida Silvestre distintas para os recifes externos e os internos. A criação de apenas uma Área de Exclusão de Uso não contemplaria todas as espécies existentes na Baía de Todos os Santos. Entretanto apenas a criação das Zonas de Preservação da Vida Silvestre não irá garantir a conservação e a recuperação dos recifes. Isso está condicionado, também, à melhoria da qualidade do ambiente interno da BTS, salientando-se o combate a pesca predatória com explosivos e redes de espera sobre os recifes, assim como a criação de áreas de exclusão de pesca, o que está condicionado a uma gestão adequada desta
Área de Proteção Ambiental após sua implantação.

\section{AGRADECIMENTOS}

À Fundação de Amparo à Pesquisa do Estado da Bahia (FAPESB) pela bolsa de mestrado e auxilio financeiro à pesquisa (Projeto Z. Leão $n^{\circ}$ ET 31/ 2004). Ao Projeto Monitoramento Ambiental de Áreas de Risco a Derramamento de Petróleo e seus Derivados - Rede05 $\backslash 01$ (PETRORISCO). À Dra. Leila de Lourdes Longo pela identificação do zoanthideo E pizoanthus sp.. Ao Prof. Dr. Francisco Barros pela auxilio na analise estatística. Aos membros da equipe que foram para o campo, Camila Brasil, Ricardo Domingues, Carlos Valerio Mendonça Filho, Eduardo Marocci, Miguel Loiola e Amanda Ercilia Carvalho, e a Rafael Carvalho que, além de participar dos trabalhos de campo, contribuiu com a confecção do mapa da BTS.

\section{BIBLIOGRAFIA}

Araújo, T.M.F., (1984) - M orfologia, C omposição, Sedimentologia e H istoria E volutiva do Recife de C oral da Ilha de Itaparica, Bahia. Dissertação de Mestrado, 92 p., Universidade Federal da Bahia, BA, Brasil. (não publicado).

Aronson, R.B. \& Precht, W.F. (2006) - Conservation, Precaution, and Caribbean Reefs. Coral Reefs, 25(3):441-450. (http://dx.doi.org/10.1007/ s00338-006-0122-9).

Artaza-Barrio, O.H. \& Schiavetti, A. (2007) - Análise da Efetividade do Manejo de duas Áreas de Proteção Ambiental do Litoral Sul da Bahia. Revista de $\mathrm{G}$ estão Costeira Integrada, 7(2):117-128. (disponível em: http://www.aprh.pt/rgci/pdf/ rgci7f2_4_artaza-barrios.pdf).

Assunção, P.V., Lima, V.F.S., Schiavetti, A., Moreau, M., Moraes, M.E.B. (2003) - Área de proteção ambiental municipal da península de Maraú (Bahia): uma análise da situação de gestão atual. Revista de $\mathrm{G}$ estão C osteira Integrada, 3:55-47 (disponível em http://www.gci.inf.br/ e d i c o e s_anteriores/03/ 16_manejo_unid_conservacao.pdf)

Bellwood, D.R., Hughes, T.P., Folke, C. \& Nystro, M. (2004) - Confronting the Coral Reef Crisis. N ature, 429: 827-833. (disponível em: http://www.nature.com/nature/journal/v429/ n6994/pdf/nature02691.pdf). 
Cruz, Kikuchi e L eão

R evista de G estão C osteira Integrada / Journal of Integrated C oastal Z one M anagement 9(3):3-23 (2009)

Callegari-Jacques, S.M. (2003). Bioestatística: prinápios e aplicaỗes. 256p., Editora Artmed, Porto Alegre, RS, Brasil. (ISBN-13: 9788536300924).

Carleton, C.B. \& Done, T.J. (1995). Quantitative Video Sampling of Coral Reef Benthos: Large-Scale Application. Coral Reefs, 14(1): 35-46. (disponível em: (http://dx.doi.org/10.1007/BF00304070)

Castro, C.B. \& Pires, D.O. (2001). Brazilian Coral Reefs: What we Already Know and What is Still Missing. Bulletin of Marine Science, 69(2):357-371. (disponível em: http://vnweb.hwwilsonweb.com/ hww/results/results_single_fulltext.jhtml;

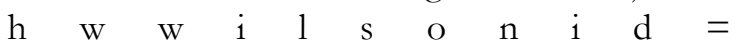
Y5RH30IVMA1KDQA3DIKSFGOADUNGIIV0).

Clake, K.R. (1993) - Nonparametric Multivariate Analyses of Changes in Community Structure. A ustralian Journal of E cology, 18:117-143.

Clake, K.R. \& Warwick, R.M. (2001). Changes in M arine Communities: A n A pproach to Statiscal A nalysis an Interpretation. 234p., Plymouth Marine Laboratory, Plymouth, Reino Unido. (ISBN-13: 978 1855311404)

Cruz, I.C.S. (2008) - Recifes de C orais da Baía deT odos os Santos, Caracterização, A valiação eIdentificação deÁ reas Prioritárias para Conservação. Dissertação de Mestrado, 102 p., Universidade Federal da Bahia, $\mathrm{BA}$, Brasil. (não publicado).

Cruz, I.C.S., Kikuchi, R.K.P. \& Leão, Z.M.A.N. (2008) - Use of Video Transect Method for Characterizing the Itacolomis Reefs, Eastern Brazil. Brazilian Journal of 0 ceanography, 56(4):271280. (disponível em: http://www.scielo.br/pdf/ bjoce/v56n4/a02v56n4.pdf).

Dudley, N. (ed.) (2008) - G uidelines for A pplying Protected A rea Management Categories. 86p., IUCN International Union for Conservation of Nature, Gland, Suíça. (ISBN: 978-2-8317-1086-0).

Dutra, L.X.C., Kikuchi, R.K.P. \& Leão, Z.M.A.N. (2006). Todos os Santos Bay Coral Reefs, Eastern Brazil, Revisited after 40 Years. Proceedings of the 10th International C oral R ef Symposium, Okinawa 1: 1090-1095. (disponível em: http: / / www.reefbase.org/download / download.aspx?type $=1 \&$ docid $=12425)$.

Edmunds, P.J. \& Carpenter, R.C. (2001) - Recovery of $\mathrm{D}$ iadema antillarum reduces macroalgal cover and increases abundance of juvenile corals on a
Caribbean reef. Proceedings of the $\mathrm{N}$ ational A cademy of Sciences, 98(9):5067-5071. (http://dx.doi.org/ $10.1073 /$ pnas.071524598)

Fernandes, L., Day, J., Lewis, A., Slegers, S., Kerrigan, B., Breen, D., Cameron, D., Jago, B., Hall, J., Lowe, D., Innes, J., Tanzer, J., Chadwick, V., Thompson, L., Gorman, K., Simmons, M., Barnett, B., Sampson, K., De'ath, G., Mapstone,B., Marsh, H., Possingham, H., Ball, I., Ward, T., Dobbs, K., Aumend, J.,Slater, D. \& Stapleton K. (2005) Establishing Representative No-Take Areas in the Great Barrier Reef: Large-Scale Implementation of Theory on Marine Protected Areas. Conservation Biology 19(6):1733-1744. (http://dx.doi.org/ 10.1111/j.1523-1739.2005.00302.x).

Governo do Estado da Bahia, (1999) - D ecreto de L ei do E stado da Bahia № 7.595 de 05 de Junho de 1999. Cria a Á rea de Proteção A mbiental - A PA da Baía de Todos os Santos edá outras providências. Publicado no Diário Oficial do Estado em 09.06.99, Salvador, BA, Brasil. (disponível em: http: / / www.meioambiente.ba.gov.br/ DecretosUnidadesdeConservacao/Dec7595.pdf). Halpern, B.S. (2003) - The Impact of Marine Reserves: Do Reserves Work and Does Reserve Size Matter? E cological A pplications, 13(1) Supplement: S117-S137. (disponível em: http://links.jstor.org/sici?sici $=1051$ $0761 \% 28200302 \% 2913 \% 3 \mathrm{~A} 1<\mathrm{S} 117$ $\% 3$ ATIOMRD>2.0.CO\%3B2-R).

Hatje, V., Barros, F.C.R., Figueiredo, D.G. \& Santos, V.L.C.S. (2006) - Trace metal contamination and benthic assemblages in Subaé estuarine system, Brazil. Marine Pollution Bulletin, 52(8): 969-977. ( h t t p : / / dx.doi.org/10.1016/ j.marpolbul.2006.04.006).

Hodgson G. (1999) - A Global Assessment of Human Effects on Coral Reefs. Marine Pollution Bulletin 38(5): 345-355. (http://dx.doi.org/10.1016/ S0025-326X(99)00002-8).

Hubbard, D.K. (1997) - Reefs as Dynamic Systems. In: Birkeland, C. (eds.), "Life and D eath of Coral Reefs”. 560p., Chapman \& Hall, New York, USA.(ISBN 13: 9780412035418$)$.

IBGE - Instituto Brasileiro de Geografia e Estatística (s/d) - IBG E Cidades - Salvador - Contagem da População 2007. (disponível em: 
Cruz, Kikuchi e L eão

R evista de G estão C osteira Integrada / Journal of Integrated C oastal Z one M anagement 9(3):3-23 (2009)

http://www.ibge.gov.br/cidadesat/xtras / perfil wind ow.ph p ? n o m e m u n = Salvador\&codmun $=292740 \& r=2$

Jameson, S.C., Erdmann, M.V., Gibson Jr., G.R., Pott, K.W. (1998) - D elelopment of Biological Criteria for Coral Reef E cosystem A ssessment. 79p., United States Environmental Protection Agency,Office of Science and Technology, Health and Ecological Criteria Division, Washington, DC, USA. (disponível em: http://www.epa.gov/owow/ oceans/coral/documents/biocrit.pdf)

Jameson, S.C., Erdmann, M.V., Karr, J.R., Pott, K.W. (2001) - Charting a Course Toward Diagnostic Monitoring: A Continuing Review of Coral Reef Attributes and a Research Strategy for Creating Coral Reef Indexes of Biotic Intedrity. Bulletin of Marine Science 69(2):701-744. (disponível em: http:/ /vnweb.hwwilsonweb.com/hww/results / getResults.jhtml?_DARGS=/hww/results / results_common.jhtml.21).

Khalaf, M.A. \& Kochzius, M. (2002) - Changes in trophic community structure of shore fishes at an industrial site in the Gulf of Aqaba, Red Sea. Marine E cology Progress Series, 239: 287-299. (http://dx.doi.org/10.3354/meps239287).

Laborel, J. (1969b) - Madréporaires et Hydrocoralliaires Récifaux des Cotes Brésiliennes: Systématique, Écologie, Répartition Verticale et Géogrphique. In: Masson, E.T. (eds.), "Résultats Scientifiques de Campagnes de la "Calypso" , Cie. Boulevard Saint-Germain, Paris, França.

Laborel, J. (1970) - L es Peuplement de Madreporaires de C ôtes Tropicales du Brésil. 260p., A nnales deL 'U niversité D 'A bidjan, Serie E - II Fascicule 3, Abidjan, Costa do Marfim. (ISBN 2-7166-0216).

Leão, Z.M.A.N., Kikuchi, R.K.P. \& Testa, V. (2003) Corals and Coral Reefs of Brazil. In: Cortés, J. (eds.), Latin A merican Coral Reefs. Elsevier B.V. (ISBN-13: 978-0444513885).

Lessa G.C., Bittencourt A.C.S.P., Brichta A. \& Dominguez J.M.L. (2000) - A Reevaluation of the Late Quaternary Sedimentation in Todos os Santos Bay (BA), Brazil. A nais da A cademia Brasileira Ciências, 72(4):573-590. (disponível em: http://www.scielo.br/pdf/aabc/v72n4/ 0052.pdf).

Madin, J.S. (2005) - Mechanical limitations of reef corals during hydrodynamic disturbances. Coral Reefs, 24(4):630-635. (http:/ / dx.doi.org/10.1007/ s00338-005-0042-0).

Martins, L.K.P., Nascimento, I.A., Fillmann, G., King, R., Evangelista, A.J.A., Reandman, J.W. \& Depledge, M.H. (2005) - Lysosomal responses as a diagnostic tool for the detection of chronic petroleum pollution at Todos os Santos Bay, Brazil. Environmental Research, 99(3):387-396. (http://dx.doi.org/10.1016/j.envres.2005.01.001).

McClanahan, T.R., Marnane, M.J., Cinner, J.E. \& Kiene W.E. (2006) - A Comparison of Marine Protected Areas and Alternative Approaches to Coral-Reef Management. C urrent Biology, 16(14):1408-1413. (http://dx.doi.org/10.1016/ j.cub.2006.05.062).

McClanahan, T.R., Muthiga,N.A.,Kamukuru, A.T., Machano, H., Kiambo, K.W. (1999) - The effects of marine parks and fishing on coral reefs of northern Tanzania. Biological Conservation 89(2): 161-182. (http://dx.doi.org/10.1016/S00063207(98)00123-2).

McField, M.D., Hallock, P. \& Jaap, W.C. (2001) Multivariate Analysis of Reef Community Structure in the Belize Barrier Reef Complex. Bulletin of M arineScience, 69(2): 745-758. (disponível em: http://vnweb.hwwilsonweb.com/hww/ results/results_single_fulltext.jhtml;hwwilsonid= OIXOZFZAUGI3RQA3DIKSFGOADUNGIIV0).

McField, M. \& Krame, P., (2007) - H ealthy Reefs for $\mathrm{H}$ ealthy People: A G uide to Indicators of $\mathrm{R}$ eef $\mathrm{H}$ ealth and Social W ell-being in the M esoamerican Reef Region. 208p., Healthy Reefs for Healthy People Initiative, The Smithsonian Institution, Washington, DC, USA. (disponível em: http://healthyreefs.org/ Book_launch/Healthy_Reef_24_complete-1.pdf).

Moura, R.L., Dutra, G.F., Francini-Filho, R.B., MinteVera, C.V., Curado, I.B., Guimarães, F.J., Oliveira, R.F. \& Alves, D.C., (2007) - Gestão do Uso de Recursos Pesqueiros na reserva Extrativista Marinha do Corumbau - Bahia. In: Caminha, A.F., Prates, A.P., Duarte, A.E.M., Blanc D., Ferreira, J.L.F., Hazin, M.C., Viana, M.S., Pereira, P.M. (eds.), Á reas protegidas do Brasil. Vol. 4, Prates, A.P. (coord.), Á reas A quáticas Protegidas como Instrumento de $G$ estão Pesqueira., pp.169-181. Ministério do Meio Ambiente, Brasília, DF, Brasil. (ISBN 978-857738-077-0). 
Mumby P.J. (2006) - The Impact of Exploiting Grazers (Scaridae) on The Dynamics of Caribbean Coral Reefs. E cological A pplications, 16(2):747-769. (ht tp: / / dx.doi.org/10.1890/10510761(2006)016[0747:TIOEGS]2.0.CO;2).

Neves, E.G. (2004) - Complexo Siderastrea: espécies distintas? Significado da variabilidade do gênero Siderastrea de Blainville, 1830 (Anthozoa, Scleractinia) no Brasil. 300p., Tese de Doutorado. Universidade de São Paulo. (não publicado).

Neves, E. G., Andrade, S. C., Silveira, F. L., Solferini, V. N. (2008) - Genetic Variation and Population Structuring in two Brooding Coral Species (Siderastrea Stellata and Siderastrea Radians) from Brazil. G enetica 132: 243-254. (http://dx.doi.org/ 10.1007/s10709-007-9168-z)

Orge, M.D.R., Porsché, I.J., Costa, M.C., Lima, J.S., Soares, S.E.D. \& Justino, R. (2000) - Assessment of Oil Refinery Waste on Rhizophora mangle L. Seedling Growth in Mangroves of Todos os Santos Bay, Bahia, Brazil. A quatic E cosystem H ealth \& M anagement, 3(4):471-477. (http://dx.doi.org/ 10.1080/14634980008650683).

Pandolfi, J.M., Bradbury, R.H., Sala, E., Hughes, T.P., Bjorndal, K.A., Cooke,R.g., McArdle, D.,McClenachan,L., Newman,M.J.H., Paredes,G., Warner, R.R. \& Jackson, J.B.C. (2003) - Global Trajectories of the Long-Term Decline of Coral Reef Ecosystems. Science, 301(5635):955-958. (http://dx.doi.org/10.1126/science.1085706).

Presidência da República (2000) - L ei Federal N ⒐985, de 18 de Julho de 2000. Regulamenta 0 art. 225, § 10, incisos I, II, III eV II da Constituição Federal, institui 0 Sistema N acional de U nidades de Conservação da N atureza edá outras providências. Publicado no Diário Oficial da União de 19.7.2000, Brasília, DF, Brasil. (disponível em: http://www.planalto.gov.br/ ccivil/leis/L9985.htm).

Riegl, B. (1999) - Corals in a Non-Reef Setting in the Southern Arabian Gulf (Dubai, UAE): Fauna and Community Structure in Response to Recurring Mass Mortality. Coral Reefs, 18(1):63-73. (http://dx.doi.org/10.1007/s003380050156).

Riegl, B. (2001) - Degradation of Reef Structure, Coral and Fish Communities in the Red Sea by Ship Groundings and Dynamite Fisheries. Bulletin of Marine Scienœ, 69: (2): 595-611. (disponível em: http://vnweb.hwwilsonweb.com/hww/results / getResults.jhtml?_DARGS=/hww/results / results_common.jhtml.21).

Riegl, B. \& Luke, K.E. (1998) - Ecological Parameters of Dynamited Reefs in the Northern Red Sea and their Relevance to Reef Rehabilitation. Marine Pollution Bulletin, 37(8-12): 488-489. (http://dx.doi.org/10.1016/S0025326X(99)00104-6).

Riegl, B. \& Piller, W.E. (1999) - Coral Frameworks Revisited-Reefs and Coral Carpets in the Northern Red Sea. Coral Reefs, 18(3): 241-253. (http://dx.doi.org/10.1007/s003380050188).

Rylands, A.B. \& Brandon, K. (2005) - Unidades de Conservação Brasileiras. Megadiversidade, 1(1):2735. (disponível em: http:/ /www.conservacao.org/ publicacoes/files/06_rylands_brandon.pdf).

Santos, V.L.C. Souza, Raymundo, C.C. \& Tavares, T. (2000) - Isomers of the dodecylbenzene in marine sediments from the Todos os Santos Bay, Bahia, Brazil. Isomers os Dodecylbenzene in Marine Sediments from the Todos os Santos Bay, Bahia, Brazil. A quatic E cosystem $\mathrm{H}$ ealth \& Management, 3(4):479-484. (http://dx.doi.org/10.1080/ 14634980008650684).

Shanks, A.L., Grantham, B.A. \& Carr, M.H. (2003) Propagule Dispersal Distance and the Size and Spacing of Marine Reserves. E cological A pplications. 13 Supplement: Marine Reserves: 159-169. (http://dx.doi.org/10.1890/1051-0761(2003) 013[0159:PDDATS]2.0.CO;2).

Silva, M. (2005a) - The Brazilian Protected Areas Program. Conservation Biology. 19(3):608-611. (http://dx.doi.org/10.1111/j.15231739.2005.00707.x).

Silva, M. (2005b) - O programa brasileiro de unidades de conservação. M egadiversidade. 1(1):22-26. (disponível em: http://www.conservacao.org/ publicacoes/files/05_Silva.pdf).

Souter, D.W. \& Lindén, O. (2000) - The Health and Future of Coral Reef Systems. 0 œan \& Coastal M anagement, 43(8-9): 657-688. (http:/ / dx.doi.org/ 10.1016/S0964-5691(00)00053-3):

Tavares, T.M., Beretta, M. \& Costa M.C. (1999) - Ratio of DDT/DDE in the All Saints Bay, Brazil and its Use in Environmental Management. Chemosphere, 38(6): 1445-1452. (http: / / dx.doi.org/ 10.1016/S0045-6535(98)00546-3). 
Venturini, N. \& Tommasi, L.R. (2004) - Polycyclic Aromatic Hydrocarbons and Changes in the Trophic Structure of Polychaete Assemblages in Sediments of Todos os Santos Bay, Northeastern, Brazil. Marine Pollution Bulletin, 48(1-2): 97-107. (http: / / dx.doi.org/10.1016/S0025326X(03)00331-X).

Wallner-Kersanach, M., Theede, H., Eversberg, U. \& Lobo, S. (2000) - Accumulation and Elimination of Trace Metals in a Transplantation Experiment with Crassostrea rhizophorae. Archives of Environmental Contamination and Toxicology. A rchives of E nvironmental Contamination and Tox icology, 38(1):40-45. (http://dx.doi.org/ $10.1007 / \mathrm{s} 002449910005)$.

Wantiez, L., Thollot, P. \& Kulbicki, M. (1997) - Effects of marine reserves on coral reef fish communities from five islands in New Caledonia. Coral Reefs 16(4): 215-224. (http://dx.doi.org/10.1007/ s003380050077).

Wasserman, J.C. \& Queiroz E.L. (2004) - The
Attenuation of Concentrations Model: A New Method for Assessing Mercury Mobility in Sediments. Q uimica N ova, 27(1):17-21. (disponível em http://quimicanova.sbq.org.br/qn/qnol/ 2004/vol27n1/03-AR02200.pdf)

West, J.M. \& Salm, R.V. (2003) - Resistance and Resilience to Coral Bleaching: Implications for Coral Reef Conservation and Management. Conservation Biology, 17(4):956-967. (http://dx.doi.org/10.1046/j.15231739.2003.02055.x).

Wilkinson, C. (ed.) (2004) - Status of Coral Reefs os the W orlds: 2004. 2 volumes, 201p + 557p., Global Coral Reef Monitoring Network, Australian Institute of Marine Science, Townsville, Queensland, Austrália. (ISSN 1447-6185). (Disponível em: vol. 1 - http://www.aims.gov.au/ pages/research / coral-bleaching/ scr2004/pdf/scr2004v1-all.pdf, vol. 2 http://www.aims.gov.au/pages/research/coralbleaching/scr2004/pdf/scr2004v2-all.pdf) 